\title{
Integrating gene delivery and gene-editing technologies by adenoviral vector transfer of optimized CRISPR-Cas9 components
}

\author{
Ignazio Maggio ${ }^{1,2} \cdot$ Hidde A. Zittersteijn ${ }^{2} \cdot$ Qian Wang $^{2} \cdot$ Jin Liü $^{2} \cdot$ Josephine M. Janssen $^{2} \cdot$ Ivan Toral Ojeda $^{2} \cdot$ \\ Silvère M. van der Maarel ${ }^{3} \cdot$ Arjan C. Lankester $^{1} \cdot \operatorname{Rob}$ C. Hoeben $\mathbb{D}^{2} \cdot$ Manuel A. F. V. Gonçalves ${ }^{2}$
}

Received: 31 July 2019 / Revised: 2 December 2019 / Accepted: 11 December 2019 / Published online: 3 January 2020

(c) The Author(s) 2020. This article is published with open access

\begin{abstract}
Enhancing the intracellular delivery and performance of RNA-guided CRISPR-Cas9 nucleases (RGNs) remains in demand. Here, we show that nuclear translocation of commonly used Streptococcus pyogenes Cas9 (SpCas9) proteins is suboptimal. Hence, we generated eCas9.4NLS by endowing the high-specificity eSpCas9(1.1) nuclease (eCas9.2NLS) with additional nuclear localization signals (NLSs). We demonstrate that eCas9.4NLS coupled to prototypic or optimized guide RNAs achieves efficient targeted DNA cleavage and probe the performance of SpCas9 proteins with different NLS compositions at target sequences embedded in heterochromatin versus euchromatin. Moreover, after adenoviral vector (AdV)-mediated transfer of SpCas9 expression units, unbiased quantitative immunofluorescence microscopy revealed 2.3-fold higher eCas9.4NLS nuclear enrichment levels than those observed for high-specificity eCas9.2NLS. This improved nuclear translocation yielded in turn robust gene editing after nonhomologous end joining repair of targeted double-stranded DNA breaks. In particular, AdV delivery of eCas9.4NLS into muscle progenitor cells resulted in significantly higher editing frequencies at defective $D M D$ alleles causing Duchenne muscular dystrophy (DMD) than those achieved by AdVs encoding the parental, eCas9.2NLS, protein. In conclusion, this work provides a strong rationale for integrating viral vector and optimized gene-editing technologies to bring about enhanced RGN delivery and performance.
\end{abstract}

\section{Introduction}

RNA-guided nucleases (RGNs) derived from prokaryotic adaptive immune systems are being repurposed for genome editing applications. Due to their ease of programmability, RGNs are revolutionizing our ability to manipulate the genome of higher eukaryote cells [1-4].

Supplementary information The online version of this article (https:// doi.org/10.1038/s41434-019-0119-y) contains supplementary material, which is available to authorized users.

Manuel A. F. V. Gonçalves

M.F.V.Goncalves@lumc.nl

1 Department of Pediatrics/Willem-Alexander Kinderziekenhuis (WAKZ), Leiden University Medical Center, Albinusdreef 2, 2300 RC Leiden, The Netherlands

2 Department of Cell and Chemical Biology (CCB), Leiden University Medical Center, Einthovenweg 20, 2333 ZC Leiden, The Netherlands

3 Department of Human Genetics, Leiden University Medical Centre, Einthovenweg 20, 2333 ZC Leiden, The Netherlands
Among an increasing number of orthologs, the most commonly used RGNs are derived from the type II clustered, regularly interspaced, short palindromic repeats (CRISPR)associated Cas9 (CRISPR-Cas9) system from Streptococcus pyogenes [5-7]. These RGNs work as a bipartite molecular scissor whose components are a Cas9 nuclease and a chimeric guide RNA (gRNA) [5-7]. The gRNA is an engineered single transcript consisting of a sequence-tailored CRISPR RNA (crRNA) linked to a transactivating crRNA (tracrRNA) moiety $[1,5,6]$. The $5^{\prime}$-end of the crRNA (spacer) directs specificity by binding to a DNA sequence through Watson-Crick base pairing. However, prior to crRNA hybridization to the target sequence, Cas9 needs to recognize a short protospacer adjacent motif (PAM), whose sequence is NGG in the case of $S$. pyogenes Cas9 (SpCas9). Hence, a 20-nucleotide (nt) sequence complementary to genomic DNA positioned next to a PAM defines a canonical target site for RGN complexes [1, 5-7].

After binding to the target site, the Cas9 nuclease undergoes conformational changes that lead to the activation of its two nuclease domains (i.e., RuvC and $\mathrm{HNH}$ ) with subsequent generation of a double-stranded DNA break 
(DSB) [8, 9]. In somatic mammalian cells, DSBs are deleterious genomic lesions resolved by endogenous DNA repair pathways, most often through the nonhomologous end joining (NHEJ) pathway. The repair of RGN-induced DSBs by NHEJ can yield small insertions and deletions (indels) at predefined genomic positions. These indels can be exploited for functional knockout of genes and/or DNA motifs as well as restoration of out-of-frame coding sequences [1, 2].

Building on the extensive characterization of SpCas9 and gRNA structures [8-12], the efficiency and specificity of RGN-mediated genome editing are steadily improving by rationally designing individual CRISPR-Cas9 components or by directed protein evolution [4]. For instance, mutations in the PAM-interacting regions are the basis for SpCas9 variants with alternative PAM specificities [13, 14]. Furthermore, the amino acids substitutions N497A/R661A/ Q695A/Q926A and N692A/M694A/Q695A/H698A have generated the high-specificity SpCas9-HF1 and HypaCas9 variants, respectively $[15,16]$. These mutations are localized within REC3, a noncatalytic domain of SpCas9 involved in RNA-DNA heteroduplex recognition and in conformational activation of the $\mathrm{HNH}$ nuclease domain $[15,16]$. Hence, by interfering with proofreading activity, the aforementioned alanine mutations in SpCas9-HF1 and HypaCas9 heighten the threshold for conformational $\mathrm{HNH}$ activation leading to increased specificity over wild-type SpCas9 [15, 16]. In another study, substitutions of residues interacting with the nontarget DNA strand (i.e., K848A/ K1003A/R1060A) were shown to confer high specificity to the eSpCas9(1.1) variant [17]. In side-by-side comparisons, eSpCas9(1.1) tend to display higher on-target activities than those of SpCas9-HF1 [16, 18, 19].

Parallel efforts are also being devoted to improving the gRNA component. For instance, truncating the $5^{\prime}$-end of gRNAs by a few nts (Tru-gRNAs), can reduce off-target RGN cutting activity [20]. Furthermore, mutations disrupting a cryptic RNA polymerase III (Pol-III) terminator combined with the extension of the duplex located between the crRNA and tracrRNA moieties, have yielded optimized gRNA scaffolds which can improve RGN performance, presumably by increasing gRNA expression and stability in target cells [21, 22].

Despite constant efforts to optimize CRISPR-Cas9 components, further improvements on intracellular delivery and target DNA cleavage remain in demand for advancing genome editing in human cells [2, 23]. Here, to address these requirements, we assembled RGNs containing optimized SpCas9 and gRNA components. In particular, we investigated the effects on nuclear localization and targeted DSB frequencies of RGNs containing the high-specificity eSpCas9(1.1) nuclease with two or four nuclear localization signals (NLSs). NLSs are small peptides that mediate the nuclear import of cargo molecules to which they are either linked in artificial constructs or present in native proteins [24, 25]. Moreover, we tested the feasibility of coupling gRNAs harboring optimized scaffolds [22] to eSpCas9(1.1) with two or four NLSs. We report that endowing the highspecificity eSpCas9(1.1) nuclease [17], hereinafter named eCas9.2NLS, with two extra NLSs (eCas9.4NLS) improves protein nuclear compartmentalization, ultimately leading to enhanced targeted DSB formation. To further extend the delivery and hence applicability of these tools, we packaged the large expression units encoding SpCas9 variants in second-generation adenoviral vectors $(\mathrm{AdVs})$. In contrast to other recombinant viral systems, e.g., lentiviral and adenoassociated viral vectors, AdVs have a strict episomal nature and large packaging capacity permitting high-level transitory expression of sizable RGN-encoding transgenes, both in vitro and in vivo [26-30].

\section{Results}

\section{Construct designs for improving RGN performance in human cells}

We started by transfecting HeLa cells with Cas9 or eCas9.2NLS plasmids. The Cas9 construct contains a human codon-optimized $\operatorname{SpCas} 9$ open reading frame (ORF) linked to a SV40 large T antigen (SV40) NLS sequence [5], whereas the eCas9.2NLS construct contains the eSpCas9 (1.1) ORF flanked by the SV40 and nucleoplasmin NLS sequences [17] (Fig. 1a). In this study, in which the performances of high-specificity eCas9.2NLS and eCas9.4NLS are compared, the regular prototypic Cas 9 nuclease linked to a SV40 NLS served as a reference [5]. Thus, although each of these individual nucleases were assessed (i.e., Cas9, eCas9.2NLS and eCas9.4NLS), conclusions regarding the effect of extra NLSs are only drawn from comparing parental eCas9.2NLS with its eCas9.4NLS derivative (Fig. 1a).

Initial transfection experiments on HeLa cells followed by immunofluorescence microscopy analysis showed that substantial amounts of Cas9 and eCas9.2NLS proteins were located in the cytoplasm (Supplementary Fig. 1). Seeking to enhance the nuclear enrichment of high-specificity eCas9.2NLS, we generated eCas9.4NLS by adding two SV40 NLS coding motifs at the terminus of the eCas9.2NLS ORF (Fig. 1a). Moreover, to investigate whether different gRNA structures can impact the genome editing efficiencies when combined with high-specificity SpCas9 nucleases, we tested gRNAs with standard or optimized scaffolds (optgRNAs) (Fig. 1a). As aforementioned, the opt-gRNAs have a tracrRNA with an extended loop and a point mutation disrupting a cryptic RNA Pol-III pause signal [22, 31]. In side-by-side comparisons with conventional gRNAs, 
A

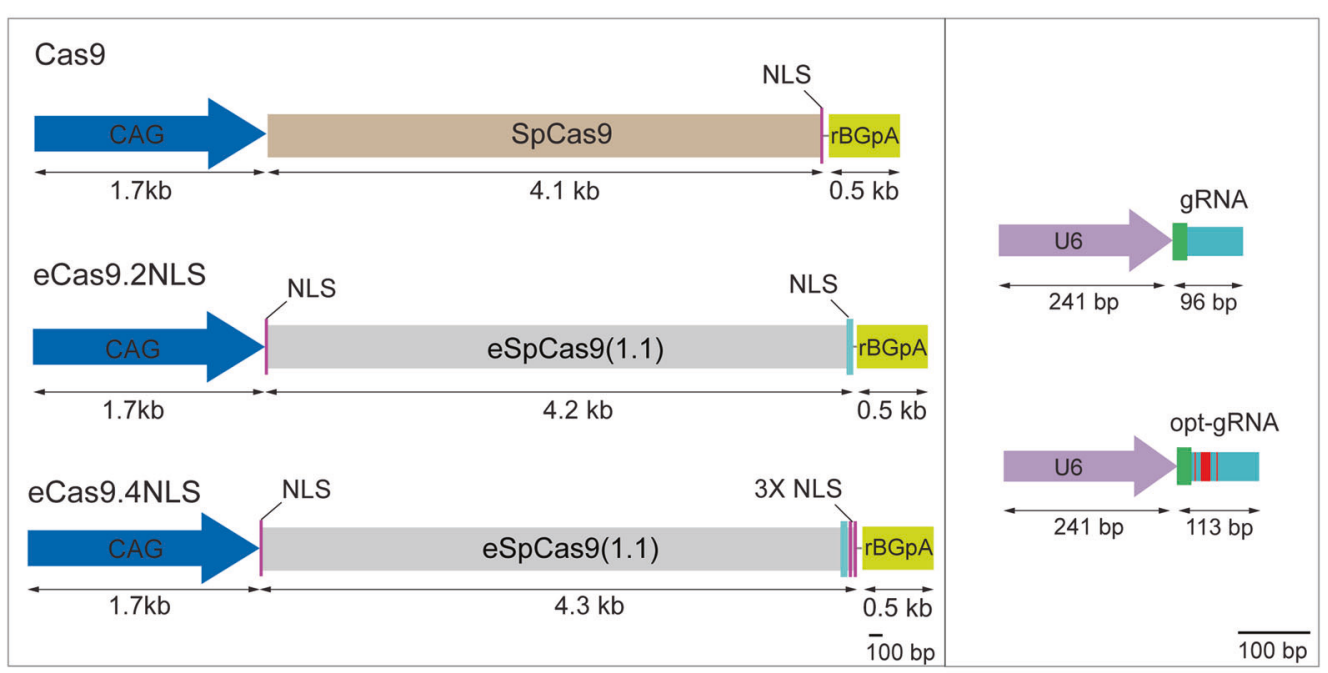

B
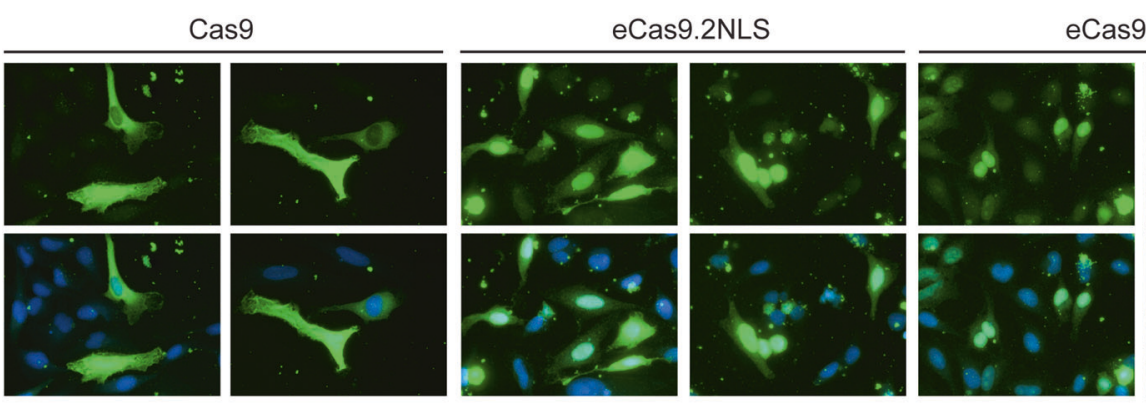

Cas 9.4 NLS
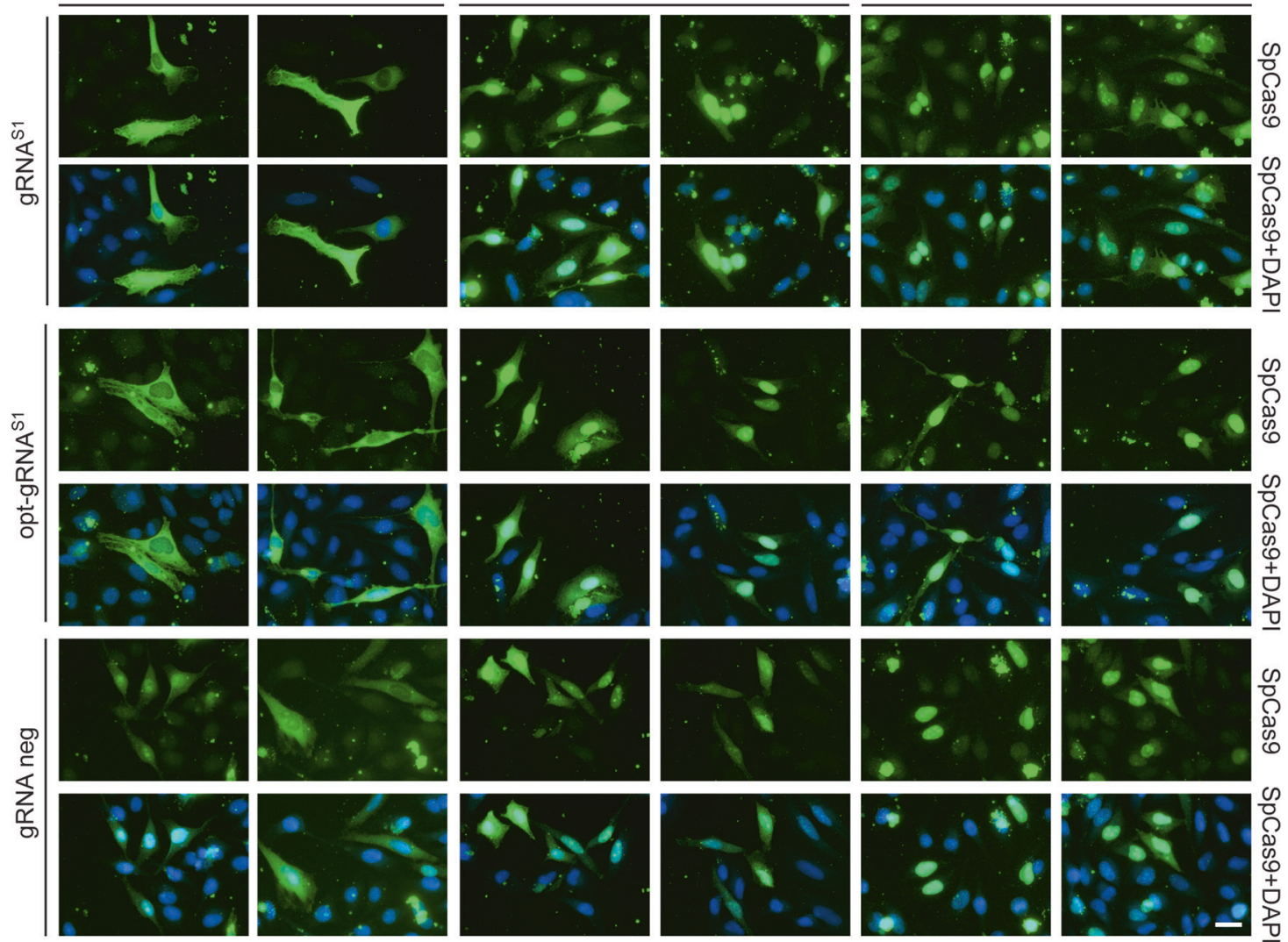

Fig. 1 Schematics of the genome editing tools and assessment of cellular nuclease distribution. a Schematic representation of the constructs deployed to express the CRISPR-Cas9 components. The hybrid CAG promoter is composed of sequences from the cytomegalovirus (CMV) immediate-early enhancer, the first exon/intron of the chicken $\beta$-actin gene, and the splice acceptor of the rabbit $\beta$-globin gene. Magenta stripes, nuclear localization signal (NLS) from the SV40 large T antigen; cyan stripes, NLS from the nucleoplasmin of Xenopus sp.; rBGpA, rabbit $\beta$-globin polyadenylation signal; U6, RNA Pol-III promoter from the human U6 gene; opt-gRNA, gRNA

with an optimized tracrRNA; red stripes, nt differences between conventional gRNA and opt-gRNA scaffolds. b SpCas9 immunofluorescence microscopy on HeLa cells. HeLa cells transfected with the indicated combinations of constructs were stained for SpCas9. Two representative fluorescence microscopy images for each experimental condition were acquired at 5 days post transfection. The staining for SpCas9 proteins and DNA was performed with an antibody specific for the C-terminus of SpCas9 and DAPI, respectively. The white horizontal bar corresponds to $30 \mu \mathrm{m}$. 
opt-gRNAs were shown to improve knockout efficiencies at CCR5 and CD4 when combined with regular SpCas9 [22]. Here, we probed the compatibility of opt-gRNAs with highspecificity eCas9.2NLS and the new eCas9.4NLS variant.

The expression of Cas9, eCas9.2NLS and eCas9.4NLS was assessed by transient transfection of HeLa cells. In this experiment, each of the nuclease expression constructs was cotransfected with plasmids encoding $\mathrm{gRNA}^{\mathrm{S} 1}$ or optgRNA $^{\mathrm{S} 1}$ or, as negative controls, with plasmids lacking gRNA expression cassettes (i.e., gRNA neg). The gRNA ${ }^{\mathrm{S} 1}$ and opt-gRNA ${ }^{\mathrm{S} 1}$ molecules address the nucleases to the human AAVS1 "safe harbor" locus at 19q13.42, and differ exclusively in that they have conventional and opt-gRNA scaffolds, respectively. Immunostaining for SpCas9 proteins followed by fluorescence microscopy analysis of transfected HeLa cells demonstrated a differential extent of nuclear localization between the different proteins with an increasing trend toward nuclear accumulation observed for those with two or four NLSs (Fig. 1b). This trend was similar independently of the type of gRNA used (Fig. 1b).

\section{Targeted AAVS1 cleavage with optimized CRISPR- Cas9 components}

Next, we sought to investigate the potential of combining eCas9.2NLS and eCas9.4NLS with opt-gRNAs for achieving efficient on-target DNA cleavage. To this end, we targeted the AAVS1 locus owing to its common use as a "safe harbor" for achieving stable and homogeneous transgene expression in cell populations [32]. In these experiments, we exposed Hela cells to RGNs consisting of Cas9, eCas9.2NLS or eCas9.4NLS partnered with gRNA ${ }^{\text {S1 }}$ or opt-gRNA ${ }^{\mathrm{S} 1}$ (Fig. 2a). As detected by T7 endonuclease I (T7EI)-based genotyping assays, eCas9.2NLS and eCas9.4NLS led to efficient NHEJ-derived indel formation at AAVS1 when combined with opt-gRNA ${ }^{\mathrm{S} 1}$ (Fig. 2b). Importantly, the highest indel frequencies were achieved by combining eCas9.4NLS with opt-gRNA ${ }^{\mathrm{S} 1}$ (Fig. 2b).

\section{Assessing optimized CRISPR-Cas9 components in loss-of-function cellular systems}

For a more sensitive assessment of RGN activities, we performed gene knockout experiments targeting $e G F P$ or $m$ Turquoise 2 reporter genes. We started by targeting two different sequences in $e G F P$ by deploying opt-gRNA ${ }^{\text {eGFP.16 }}$ and opt-gRNA ${ }^{\text {eGFP.6 }}$ (Fig. 3a, b and Supplementary Table 1). Gene knockout levels mediated by opt-gRNA ${ }^{\text {eGFP.16 and }}$ opt-gRNA ${ }^{\text {eGFP.6 }}$ were compared with those achieved by gRNA ${ }^{\text {eGFP.16 }}$ and gRNA ${ }^{\text {eGFP.6 }}$ containing the conventional scaffold (Fig. 3a, b and Supplementary Table 1). The gRNA expression plasmids were cotransfected with Cas9, eCas9.2NLS or eCas9.4NLS constructs into H27 cells.
A

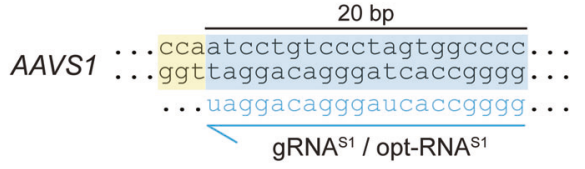

\section{B}

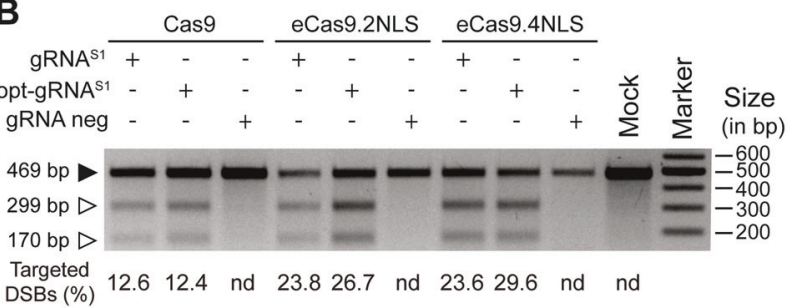

Fig. 2 Detection of targeted DSB formation at AAVS1 in HeLa cells. a Target site composed of gRNA-matching sequence (protospacer) and PAM (light blue and yellow boxes, respectively). This "safe harbor" AAVS1 locus sequence locates within the first intron of the human PPPIR12C gene (19q13.42). b Detection of AAVS1-targeted DSB formation. Genomic DNA samples from Hela cells transfected with the indicated constructs were subjected to T7EI-based genotyping assays at 3 days post transfection. Cells transfected with plasmids lacking gRNA expression cassettes (gRNA neg) or mocktransfected provided for negative controls. Open and solid arrowheads indicate the positions of DNA species corresponding to digested and nondigested amplicons, respectively. Marker, GeneRuler DNA Ladder Mix; nd not detected.

The $\mathrm{H} 27$ cell line is a HeLa-derived clone containing a single-copy, constitutionally active, eGFP transcriptional unit [33]. NHEJ-mediated repair of RGN-induced DSBs introduces frame-shifting indels that lead to $e G F P$ knockout whose frequencies can be quantified by flow cytometry [28]. Transfection experiments in $\mathrm{H} 27$ cells revealed that

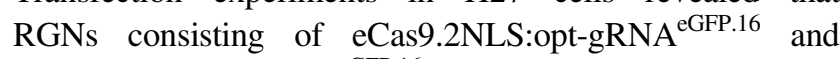
eCas9.4NLS:opt-gRNA ${ }^{\text {eGFP.16 }}$ complexes led to robust $e G F P$ knockout (Fig. 3a and Supplementary Table 1). Furthermore,

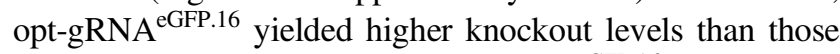
obtained by its nonoptimized gRNA ${ }^{\text {eGFP.16 }}$ counterpart (Fig. 3a and Supplementary Table 1). Similar experiments deploying instead opt-gRNA ${ }^{\text {eGFP.6 }}$ and gRNA ${ }^{\text {eGFP.6 }}$ confirmed that the eCas9.2NLS and eCas9.4NLS are compatible with the use of gRNAs with optimized scaffolds (Fig. 3b and Supplementary Table 1).

Tru-gRNAs can increase the specificity of RGNs containing the conventional SpCas9 nuclease [20]. However, combining Tru-gRNAs with eCas9.2NLS often results in reduced on-target activity [17, 34-36]. To probe the effect of combining eCas9.4NLS with truncated opt-gRNAs on targeted DNA cleavage, we generated constructs expressing gRNA $^{\text {eGFP.5 }}$ and opt-gRNA ${ }^{\text {eGFP.5 }}$ whose target site is 1-nt shorter than that of gRNA $^{\text {eGFP.6 }}$ and opt-gRNA ${ }^{\text {eGPF.6 }}$ (Supplementary Fig. 2). After transfecting H27 cells with plasmids expressing gRNA ${ }^{\text {eGFP.5 }}$ or opt-gRNA ${ }^{\text {eGFP.5 }}$ each mixed with plasmids encoding Cas9, eCas9.2NLS or eCas9.4NLS, gene knockout frequencies were again 
A

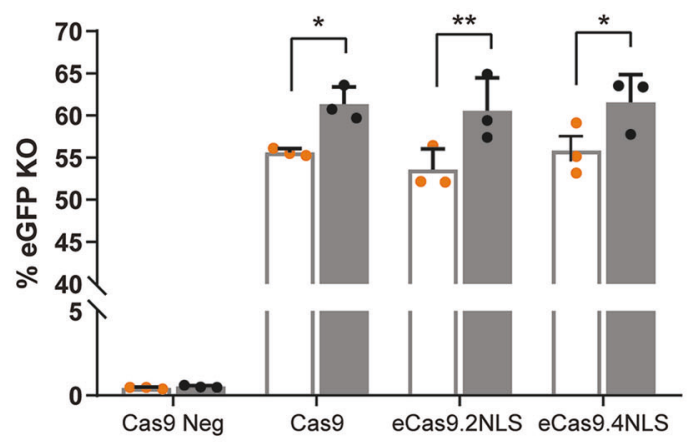

C
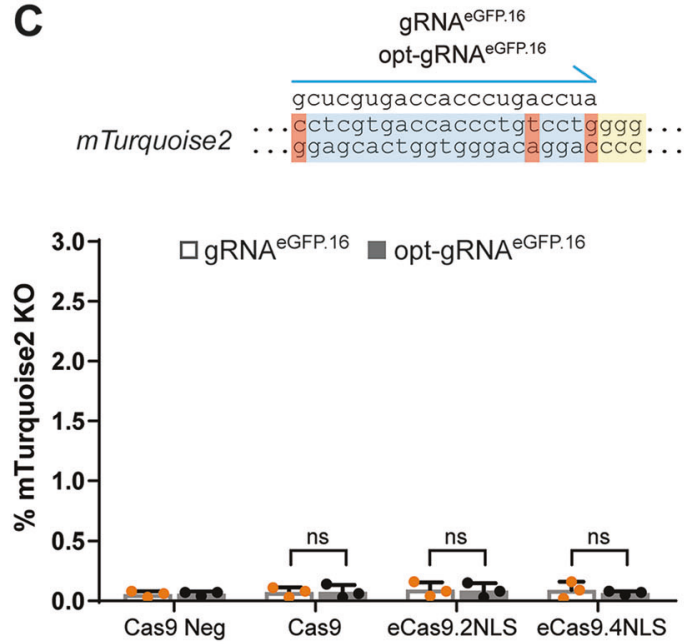

B $\quad$ GgRNA ${ }^{\text {eGFP. }}$ opt-gRNA ${ }^{\text {eGFP.6 }}$

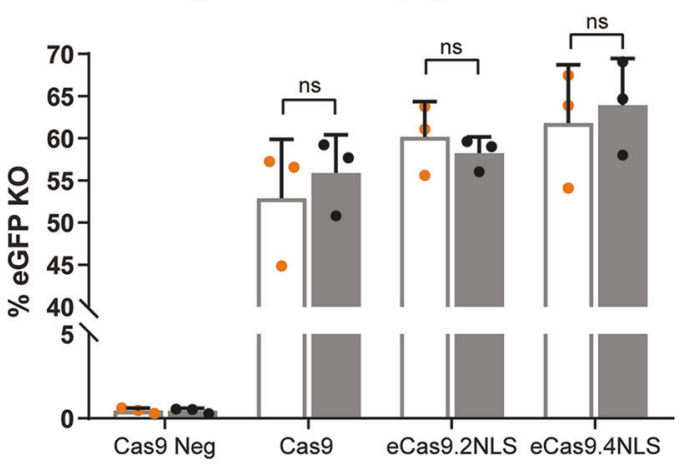

D
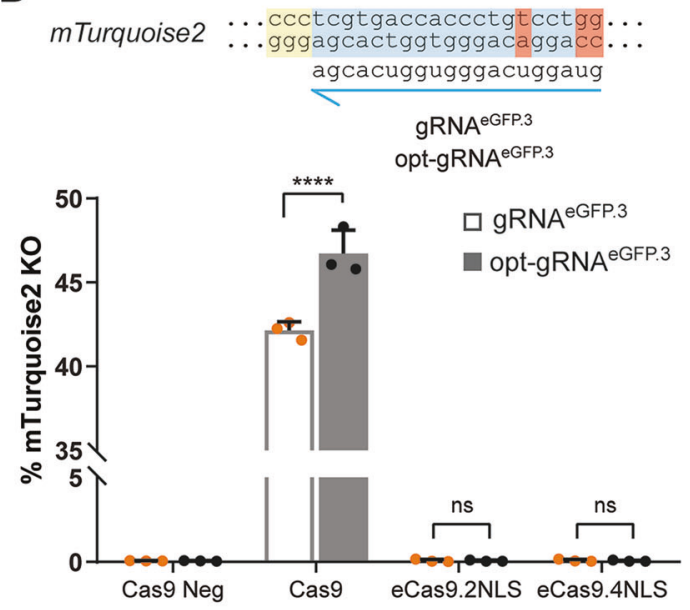

E
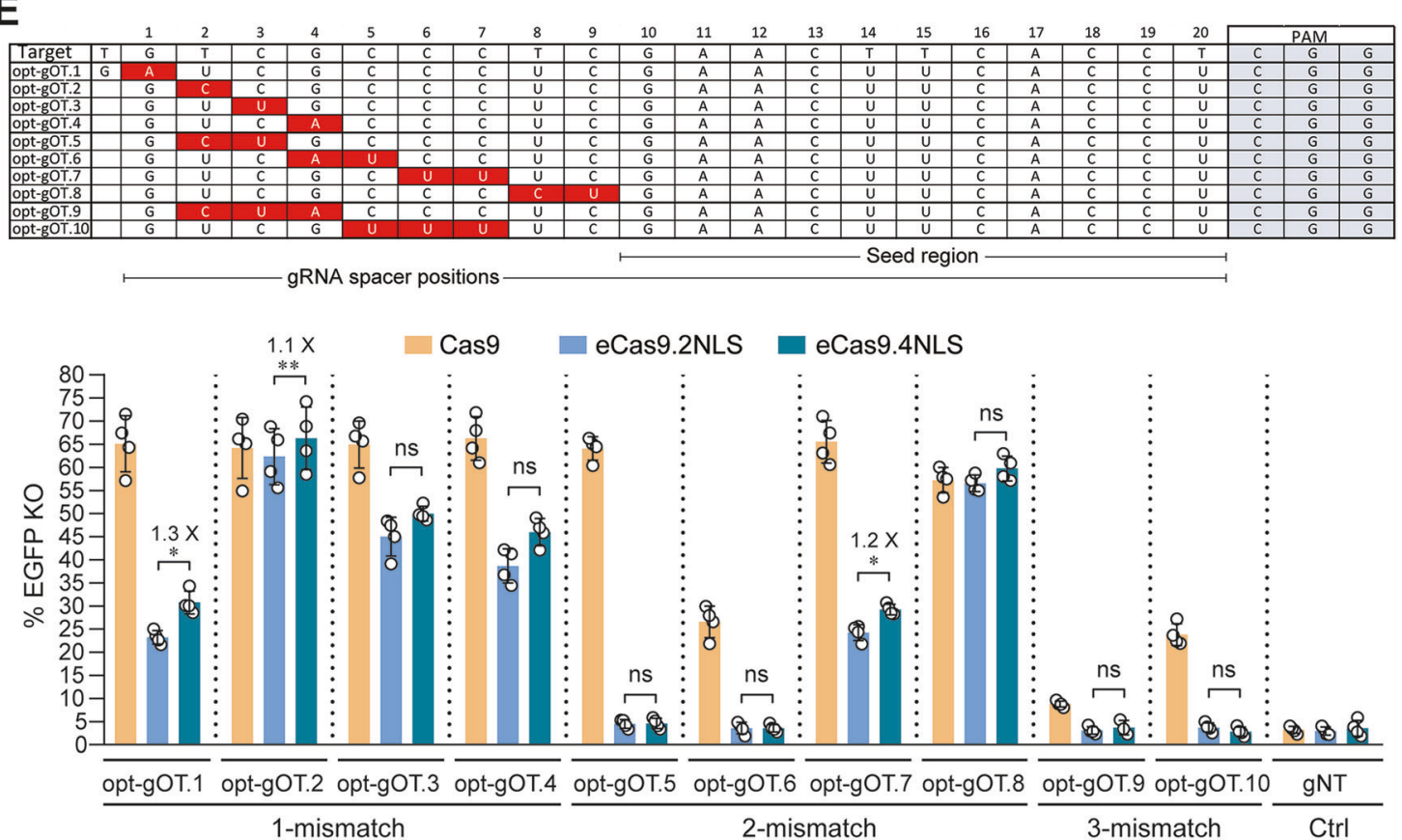

determined by flow cytometry. Expression of eCas9.2NLS: gRNA $^{\text {eGFP.5 }}$ and eCas9.4NLS:gRNA ${ }^{\text {eGFP.5 }}$ complexes led to eGFP knockout frequencies that where not higher than those obtained with Cas9:gRNA ${ }^{\text {eGFP.5 }}$ (Supplementary
Fig. 2 and Supplementary Table 2). Albeit not statistically significant, deploying opt-gRNA ${ }^{\text {eGFP.5 }}$ yielded a slight increase in target DNA cleavage when compared to that achieved with gRNA ${ }^{\text {eGFP.5 }}$, regardless of the SpCas9 partner 
Fig. 3 Evaluating the performance of optimized RGN complexes. a, b Testing the activity of optimized RGNs in reporter cells. Knockout levels in eGFP-positive $\mathrm{H} 27$ cells transfected with constructs expressing conventional or optimized RGNs. eGFP disruption frequencies were determined by flow cytometry of $\mathrm{H} 27$ cells transfected with the indicated constructs. Analysis was performed at 10 days post transfection. Bars and error bars represent mean and SD, respectively, from three independent experiments. Data significance was calculated with repeated measures one-way ANOVA followed by Tukey's test for multiple comparisons: ns nonsignificant, $P \geq 0.05$; $* P<0.05 ; * * P<0.01$. c, d Testing the specificity of optimized RGNs in mTurquoise2-positive $\mathrm{H} 11.7$ cells. Upper panel, mTurquoise 2 target sites composed of gRNA-matching sequences (protospacers) and PAMs (light blue and yellow boxes, respectively). Red boxes highlight mismatching nucleotides between gRNAs and target sites. Lower panel, mTurquoise 2 disruption frequencies determined by flow cytometry of H11.7 transfected with the indicated constructs. Analysis was performed at 10 days post transfection. Bars and error bars correspond to mean and SD, respectively, from three independent experiments. Data significance was calculated with repeated measures one-way ANOVA followed by Tukey's test for multiple comparisons: ns nonsignificant, $P \geq 0.05$; $* * * * P<0.0001$. e Testing the specificity of optimized RGNs in eGFP-positive $\mathrm{H} 27$ cells. Upper panel, sequences of opt-gRNAs opt-gOT.1 through opt-gOT.10 containing 1- 2- or 3-nt mismatches (red boxes) to a $e G F P$ sequence (target). The canonical 20-nt long gRNA spacer positions and respective 10-nt long seed region, are delimited. Mismatches locating within the seed region are purportedly more detrimental to RGN activity than those present outside this region. PAM, protospacer adjacent motif. Lower panel, $e G F P$ knockout frequencies determined by flow cytometry of $\mathrm{H} 27$ cells transfected with the indicated plasmids. A $e G F P$ nontargeting gRNA (gNT) was used as negative control. Analysis was carried out at 10 days post transfection. Bars and error bars correspond to mean and $\mathrm{SD}$, respectively from four independent experiments (except for optgOT.9 where the bars and error bars correspond to mean and SD from three independent experiments). Significance between the indicated datasets was calculated by two-tailed Student's $t$ tests: ns nonsignificant, $P \geq 0.05 ; * P<0.05 ; * * P<0.01$.

selected (i.e., Cas9, eCas9.2NLS or eCas9.4NLS) (Supplementary Fig. 2 and Supplementary Table 2).

As RGNs consisting of opt-gRNAs and eCas9.2NLS or opt-gRNAs and eCas9.4NLS generate robust on-target DNA cleavage, we next sought to investigate whether these RGNs retain a high-specificity profile. For this purpose, an off-target RGN activity detection system based on H11.7 cells was set up. The H11.7 cell line is a HeLaderived clone whose genome contains a single copy of a constitutionally active mTurquoise 2 transcriptional unit [37]. In these experiments, H11.7 cells were exposed to Cas9, eCas9.2NLS or eCas9.4NLS together with

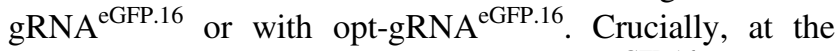
mTurquoise 2 allele, eGFP-specific gRNA ${ }^{\text {eGFP.16 }}$ and optgRNA $^{\text {eGFP.16 }}$ have 2-nt mismatches at the PAM-proximal side and 1-nt mismatch at the most PAM-distal position (Fig. 3c). Thus, these and other polymorphisms distinguishing eGFP from mTurquoise 2 turn $\mathrm{H} 11.7$ cells, together with $e G F P$-specific gRNAs, into a reporter system for off-target RGN activities through the quantification of mTurquoise2-negative populations. Flow cytometric analysis of transfected H11.7 cells revealed that the 3-nt mismatches between mTurquoise 2 and the gRNA sequences in gRNA ${ }^{\text {eGFP.16 }}$ and opt-gRNA ${ }^{\text {eGFP.16 }}$ were enough to abolish DNA cutting activity, regardless of the nuclease variant applied (Fig. 3c and Supplementary Table 1). To further probe for specificity, we took advantage of the DNA sequence flanking the off-target site of gRNA $^{\text {eGFP.16 }}$ and opt-gRNA ${ }^{\text {eGFP.16. }}$. This sequence allowed designing "inverted" gRNA ${ }^{\text {eGFP.3 } 3}$ and opt-gRNA ${ }^{\text {eGPP.3 }}$ whose DNAmatching sequence overlaps with that of gRNA ${ }^{\text {eGFP.16 }}$ and opt-gRNA ${ }^{\text {eGFP.16 }}$ (Fig. 3d). Therefore, in contrast to gRNA $^{\text {eGFP.16 } 16}$ and opt-gRNA ${ }^{\text {eGFP.16, }}$ gRNA eGFP.3 $^{\text {and opt- }}$ gRNA $^{\text {eGFP.3 }}$ have 3 -nt mismatches exclusively at the PAMdistal end of their off-target sequences (Fig. 3d). Since mismatches at the PAM-distal side are usually more tolerated than those at the PAM-proximal end [38, 39], deploying gRNA $^{\text {eGFP.3 }}$ and opt-gRNA ${ }^{\text {eGFP.3 }}$ allowed us testing off-target RGN activities under more stringent conditions than those pertaining to gRNA ${ }^{\text {eGFP.16 }}$ and optgRNA $^{\text {eGFP.16 }}$ (Fig. 3c). Exposing H11.7 cells to Cas9: gRNA $^{\text {eGFP.3 }}$ complexes readily yielded high frequencies of mTurquoise2-negative cells (Fig. 3d and Supplementary Table 1). Of note, the off-target activity of Cas9 in H11.7 cells was significantly higher when coupled to optgRNA $^{\text {eGFP.3 }}$ instead of gRNA ${ }^{\text {eGFP.3 }}$ (Fig. 3d and Supplementary Table 1). These data suggest that opt-gRNAs can increase off-target activities if combined with the native Cas9 protein. Crucially, in contrast to Cas9, neither eCas9.2NLS nor eCas9.4NLS led to detectable mTurquoise 2 disruption when coupled to $\mathrm{gRNA}^{\mathrm{eGFP} .3}$ or opt-gRNA $^{\text {eGFP.3 }}$ (Fig. 3d and Supplementary Table 1). To broaden the comparison of the specificity profiles of eCas9.2NLS versus eCas9.4NLS at different off-target sites, we generated a panel of constructs expressing opt-gRNAs (opt-gOT.1 through opt-gOT.10) with different nt mismatches to an $e G F P$ target sequence (Fig. 3e, top panel). Flow cytometric analysis of eGFP-expressing $\mathrm{H} 27$ cells [33] exposed to complexes between Cas9, eCas9.2NLS or eCas9.4NLS and each of these opt-gRNAs established that eCas9.4NLS retains most of the high specificity of its parental eCas9.2NLS protein (Fig. 3e, bottom panel and Supplementary Table 3 ). Indeed, the cumulative data gathered in Fig. 3c-e show that amongst the 12 gRNAs with mismatches tested only three conferred statistically significant higher off-target activities to eCas9.4NLS when compared to eCas9.2NLS (i.e., opt-gOT.1, opt-gOT.2, and opt. gOT.7). Importantly, these off-target activity increments associated with opt-gOT.1, opt-gOT.2, and opt-gOT.7 were low, i.e., 1.3-fold, 1.1-fold, and 1.2-fold, respectively (Fig. 3e, bottom panel and Supplementary Table 3).

The DNA binding and cleaving activities of RGNs are, to some extent, hindered at heterochromatic regions in eukaryotic cells [34, 40-44]. Hence, we next examined the 


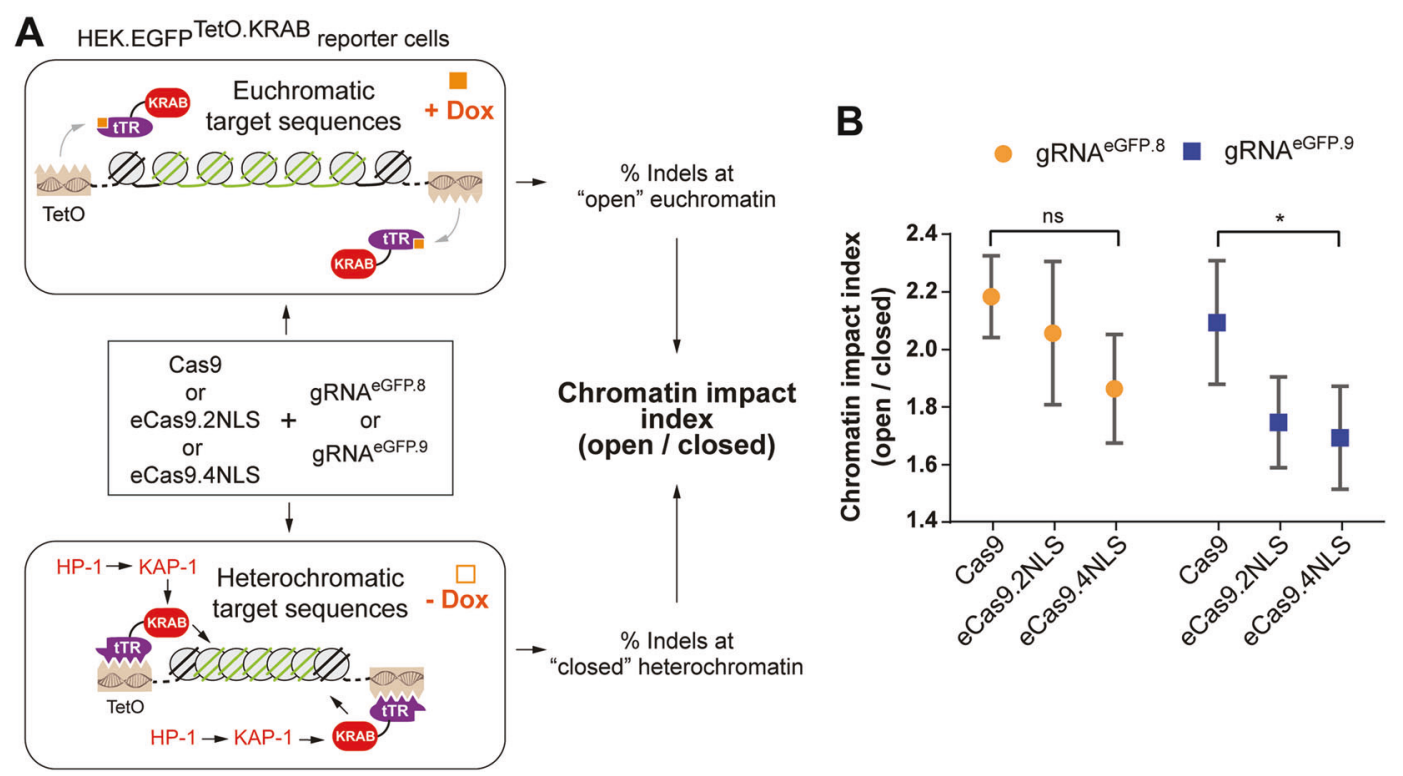

Fig. 4 Probing the effect of chromatin conformation on the activity of Cas9 nucleases with different NLS content. a Comparing the chromatin impact indexes of conventional versus optimized SpCas9 nucleases. The HEK.EGFP ${ }^{\text {TetO.KRAB }}$ reporter cells, incubated in the presence or absence of Dox, were transfected with the indicated RGN components. HEK.EGFP ${ }^{\text {TetO.KRAB }}$ cells contain single-copy $e G F P$ alleles flanked by $\mathrm{Tet} O$ elements. In the absence of Dox (-Dox), tTR$\mathrm{KRAB}$ fusion proteins bind to TetO elements and induce heterochromatin formation through the recruitment of, amongst other factors, KAP1 and HP1. In the presence of Dox, tTR-KRAB unbinds from $T e t O$ elements ultimately leading the $e G F P$ target sequences to acquire an "open" euchromatic state. To determine indel frequencies, Dox was

degree with which different chromatin structures impact the performance of Cas9, eCas9.2NLS and eCas9.4NLS. To this end, we deployed a readout system based on tTRKRAB-expressing HEK.EGFP ${ }^{\text {TetO.KRAB }}$ cells $[34,40,45]$. These cells contain single-copy $e G F P$ alleles flanked by $\mathrm{Tet} O$ elements $[34,40,45]$. In the absence of doxycycline (Dox), the binding of the tTR-KRAB fusion protein to its cognate TetO elements recruits endogenous KAP1-HP1associated epigenetic remodeling complexes that trigger heterochromatin formation at $e G F P$ sequences (Fig. 4a). In the presence of Dox, tTR-KRAB suffers a conformational change preventing it from binding to the TetO elements, ultimately leading $e G F P$ sequences to acquire an "open" euchromatic state [34, 40, 45] (Fig. 4a).

Gene knockout experiments in HEK.EGFP ${ }^{\text {TetO.KRAB }}$ cells, incubated with or without Dox, were performed by deploying plasmids encoding gRNA ${ }^{\text {eGFP.8 }}$ and gRNA ${ }^{\text {eGFP.9 }}$ (Supplementary Fig. 3a). These gRNA expression plasmids were cotransfected with Cas9, eCas9.2NLS or eCas9.4NLS constructs. After confirming that the different plasmid mixtures yielded similar transfection efficiencies (Supplementary Fig. 3b and Supplementary Table 4), the cell populations were subcultured for 10 days, during which added to all cultures (i.e., preincubated or not with Dox) prior to eGFPdirect flow cytometry. The chromatin impact indexes correspond to the ratios between $e G F P$ knockout levels at euchromatic ( + Dox) versus heterochromatic (-Dox) target sites. b Chromatin impact indexes probed in HEK.EGFP ${ }^{\text {TetO.KRAB }}$ reporter cells. HEK.EGFP ${ }^{\text {TetO.KRAB }}$ cells were transfected with constructs coding for the indicated RGN elements. eGFP knockout frequencies at euchromatic (+Dox) and heterochromatic (-Dox) target sites are presented in Supplementary Fig. 3C. Error bars indicate mean \pm SEM corresponding to three independent experiments. Data significance was calculated with repeated measures one-way ANOVA followed by Tukey's test for multiple comparisons: ns nonsignificant, $P \geq 0.05$; $* P<0.05$.

time the various RGNs were expected to induce DSBs at either euchromatic (+Dox) or heterochromatic ( - Dox) isogenic target sites. Next, all cell cultures were exposed to Dox to allow for expression and quantification of $e G F P$ knockout frequencies via flow cytometry (Supplementary Fig. 3c and Supplementary Table 5). Flow cytometric analysis confirmed the results of previous reports showing that the activity of RGNs can be hindered by KRABinduced heterochromatin [34, 40, 45] (Supplementary Fig. 3c and Supplementary Table 5). Moreover, we detected a trend toward lower ratios between the frequencies of DSB formation at euchromatic versus heterochromatic target sites (i.e., chromatin impact indexes) when RGNs contained eCas9.2NLS or eCas9.4NLS instead of Cas9 (Fig. 4b and Supplementary Table 6). These data suggest that eCas9.2NLS and eCas9.4NLS are more capable of overcoming the compact heterochromatic barrier than Cas9.

\section{AdV-mediated delivery of SpCas9 nucleases and assessment of their subcellular localization}

The size of the eCas9.4NLS expression unit (i.e., $\sim 6.5 \mathrm{~kb}$; Fig. 1a), exceeds the effective packaging capacity of 
commonly used viral vectors, such as lentiviral and adenoassociated viral vectors [27]. Hence, to achieve efficient delivery of transcriptional units encoding optimized Cas9 nucleases into a broad range of dividing and nondividing target cells, we investigated the suitability of AdVs as delivery vehicles [26-28]. Therefore, we generated CD46targeted second-generation (i.e., E1- and E2A-deleted) AdV.Cas9, AdV.eCas9.2NLS and AdV.eCas9.4NLS. We started by transducing HeLa cells with equal doses of these SpCas9-encoding AdVs either alone or in combination with AdV.gRNA ${ }^{\mathrm{S} 1}$. The proportion between the multiplicity of infections (MOIs) of AdVs expressing SpCas9 nucleases and AdVs expressing gRNAs in this, and subsequent experiments, was $1: 1$. AdV.gRNA ${ }^{\mathrm{S} 1}$ is equally a CD46targeted second-generation AdV that expresses a gRNA addressing SpCas9 nucleases to the human AAVS1 locus [28]. Immunofluorescence microscopy analysis of SpCas9 expression showed a subcellular distribution reminiscent of that obtained after transfecting plasmids encoding the same RGNs (Figs. 1b and 5a). In particular, unbiased quantification of fluorescence intensities in SpCas9-stained cells revealed higher amounts of antigen in the nuclear compartment over the cytoplasmic compartment in cells exposed to AdV.eCas9.4NLS when compared with those present in these compartments in cells transduced with AdV.Cas9 or AdV.eCas9.2NLS (Fig. 5b, c, Supplementary Figs. 4 and 5a and Supplementary Table 7). This robust effect on the differential distribution of Cas9, eCas9.2NLS and eCas9.4NLS between the nucleus and cytoplasm of transduced cells, was similar in cell cultures incubated with or without AdV.gRNA ${ }^{\mathrm{S} 1}$ (Fig. 5b, c, Supplementary Figs. 4 and 5a and Supplementary Table 7). Importantly, enrichment in the nuclear compartmentalization of SpCas9 proteins, calculated as the ratio between geometric mean intensity (GMI) in the nucleus versus GMI in the whole cell, increased up to 14-fold by using AdV.eCas9.4NLS (Fig. 5c, Supplementary Fig. 5b and Supplementary Table 7). These data were confirmed by an independent dose-response experiment in which HeLa cells were transduced with different amounts of AdV.eCas9.4NLS mixed with or without AdV.gRNA ${ }^{\text {S1 }}$ (Supplementary Fig. 6). Indeed, in cells stained positive for nuclease expression, the SpCas9 nuclear enrichment was independent of the MOI applied (Supplementary Fig. 6). These results suggest that the extent of nuclear translocation is an intrinsic property of the nuclease which is independent of its total concentration in the cell. Finally, this quantitative single cell-level fluorescence microscopy analysis in which isogenic constructs eCas9.2NLS and eCas9.4NLS were compared side-by-side, demonstrates a clear direct relationship between the number of NLS motifs and the nuclear localization of the respective proteins (Fig. 5b, c, Supplementary Figs. 4 and 5a).

\section{Quantification of targeted DNA cleavage induced by AdVs encoding SpCas9 nucleases}

Functional validation of AdV.eCas9.4NLS was initiated by transducing HeLa cells in combination with AdV.gRNA ${ }^{\mathrm{S1}}$. T7EI-based genotyping assays revealed a dose-dependent increase in indel frequencies at AAVS1 (Supplementary Fig. 7). To accurately quantify differences in targeted DNA cleaving activities induced by AdV.Cas9, AdV. eCas9.2NLS and AdV.eCas9.4NLS, we transduced eGFPpositive $\mathrm{H} 27$ cells at different doses together with AdV. gRNA $^{\text {eGFP }}$ [28] and determined $e G F P$ knockout frequencies by flow cytometry (Fig. 6a and Supplementary Table 8). AdV.gRNA ${ }^{\text {eGFP }}$ is a CD46-targeted second-generation AdV that expresses a canonical gRNA addressing SpCas9 nucleases to the $e G F P$ reporter (Fig. 6a) [28]. At 10 days post transduction, flow cytometry and direct fluorescence microscopy revealed a clear dose-dependent increase in the fraction of eGFP-negative cells in all cultures exposed to AdV.gRNA ${ }^{\text {eGFP }}$ in combination with AdV.Cas9, AdV. eCas9.2NLS or AdV.eCas9.4NLS (Fig. 6a, Supplementary Fig. 8 and Supplementary Table 8). Crucially, at MOIs of 6 and $20 \mathrm{IU} / \mathrm{cell}$, AdV.eCas9.4NLS mediated higher $e G F P$ knockout levels when compared with those triggered by AdV.Cas9 or AdV.eCas9.2NLS (Fig. 6a and Supplementary Table 8 ).

Finally, we set out to investigate the potential of AdVs encoding eCas9.4NLS for genome editing in difficult-totransfect cells. To this end, we deployed myoblasts isolated from patients with the muscle-wasting disorder Duchenne muscular dystrophy (DMD). In particular, DMD.48-50 $0^{\mathrm{gE} 51.2}$ and DMD.45-52 $2^{\mathrm{gE} 53}$ myoblasts whose dystrophin-encoding $D M D$ gene lacks exons 48 through 50 and exons 45 through 52 , respectively [46]. These deletions destroy the $D M D$ reading frame, severally reducing dystrophin amounts in striated muscle cells (Supplementary Fig. 9). Furthermore, DMD.48-50 ${ }^{\mathrm{gE} 51.2}$ and DMD.45-52 $2^{\mathrm{gE} 53}$ myoblasts express gRNAs targeting SpCas9 nucleases to exon 51 and exon 53, respectively [46]. After AdV-mediated transfer of SpCas9 proteins into DMD.48-50 ${ }^{\text {gE51.2 }}$ and DMD. $45-52^{\text {gE53 }}$ myoblasts, the resulting RGNs can lead to the NHEJ-mediated restoration of the $D M D$ reading frame (Supplementary Fig. 9). Of note, gE51.2 and gE53 are noncanonical gRNAs in that they have a $5^{\prime}$-end-mismatching $\mathrm{G}$ and a 19 -nt spacer, respectively (Fig. 6b, c).

DMD.48-50 $0^{\text {gE51.2 }}$ myoblasts were transduced with AdV. Cas9, AdV.eCas9.2NLS, or AdV.eCas9.4NLS at MOIs of $2.5,5$, and $10 \mathrm{IU} / \mathrm{cell}$. Possibly due to the mismatching $\mathrm{G}$ in gE51.2, AdV.Cas9 led to higher indel frequencies at $D M D$ exon 51 than AdV.eCas9.2NLS as determined by T7EI-based genotyping assays (Fig. 6b and Supplementary Fig. 10). Indeed, as aforementioned, compared with the conventional Cas9, these gRNA modifications often reduce DNA cleaving 
Fig. 5 Quantification of

SpCas9 intracellular

distribution in AdV-

transduced HeLa cells.

a Representative

immunofluorescence

microscopy images of HeLa

cells transduced with the

indicated AdVs. The staining for

SpCas9 nucleases and DNA was

done at 5 days post transduction

using an antibody specific for

the C-terminus of SpCas9 and

DAPI, respectively. The

$30 \mu \mathrm{m}$. b Mean fluorescence

intensity (MFI) of SpCas9-

specific signals in the nucleus

versus cytoplasm of AdV-

correspond to the mean of

nuclear or cytoplasmic SpCas9-

derived fluorescence intensities

normalized, on a per-cell-basis,

to the whole cellular error bars indicate mean and SD, respectively, of three

independent experiments.

Significance between the

indicated datasets was calculated

by two-tailed Student's $t$ tests;

$* P<0.05$. c SpCas9 nuclear

enrichment. Values are

calculated by normalizing the geometric mean intensity (GMI) in the nucleus versus the GMI in indicate mean and SD,

respectively, of three

independent experiments.

Significance between the indicated datasets was calculated by two-tailed Student's $t$ tests; $* P<0.05$. Data were acquired with Xcyto 10 using $20 \times$ magnification. Automated cell segmentation was performed with XcytoView software. horizontal bar corresponds to

transduced cells. Values

fluorescence intensity. Bars and whole cell. Bars and error bars

A
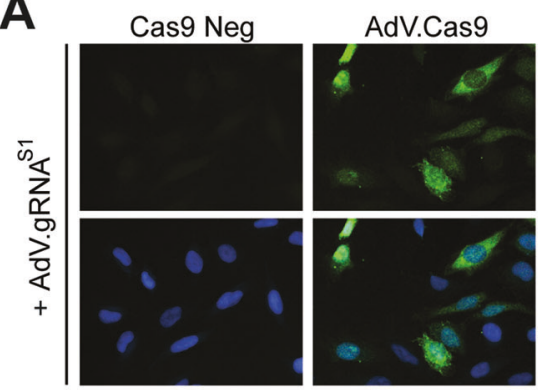

AdV.eCas9.2NLS AdV.eCas9.4NLS
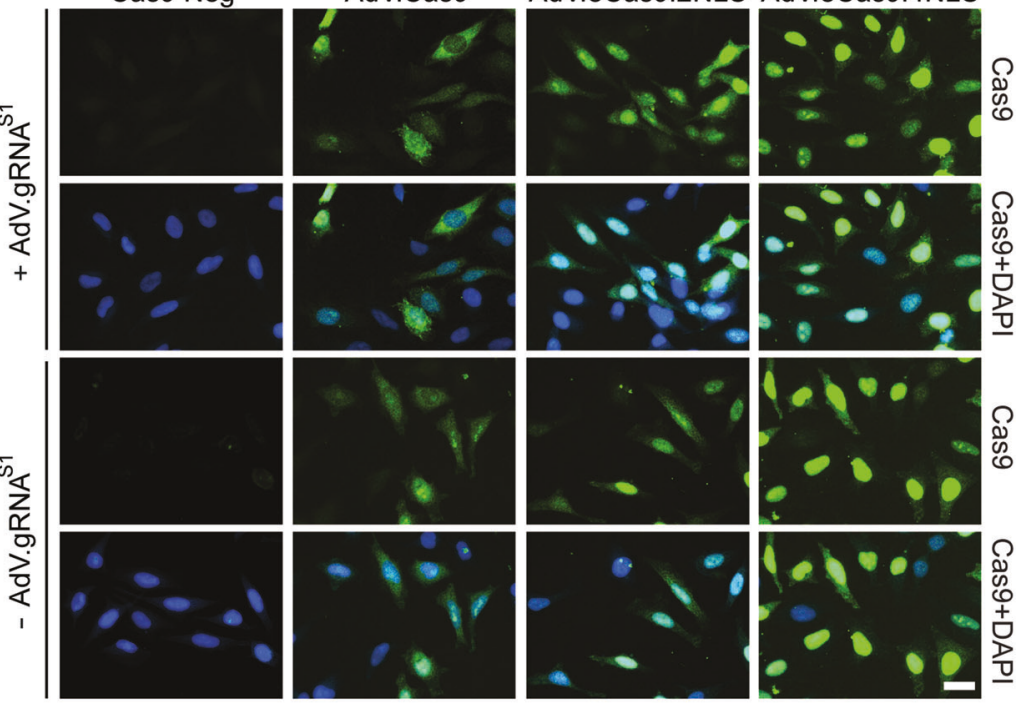

B

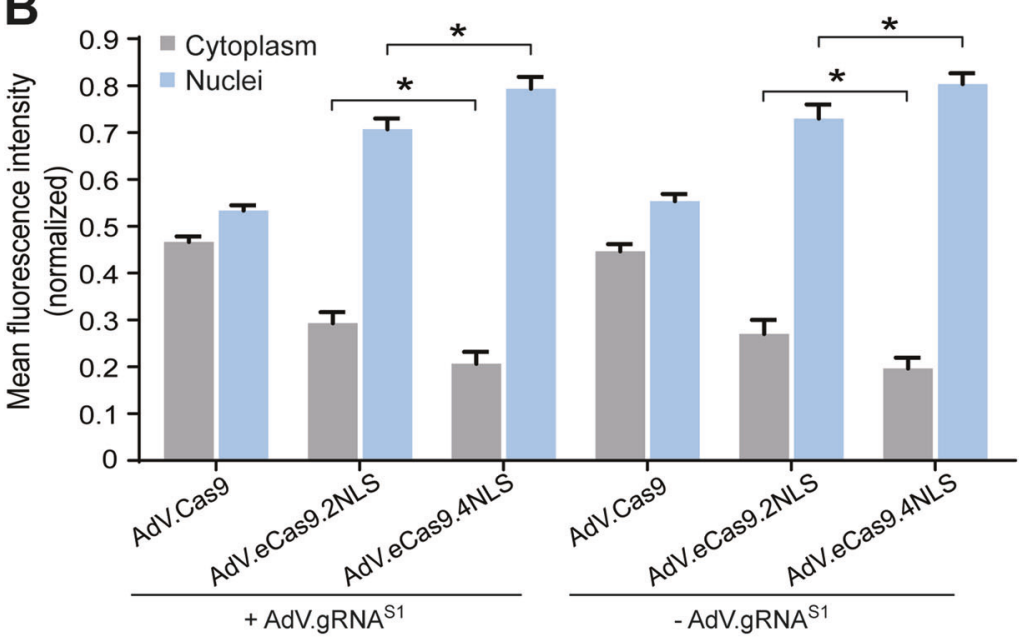

C

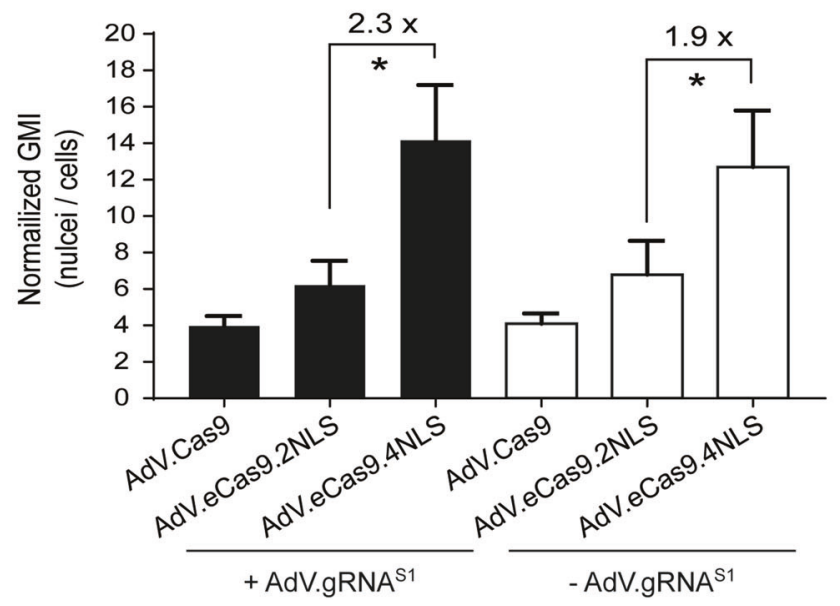

activities when coupled to high-specificity SpCas9 variants, including eCas9.2NLS [16, 34-36]. Importantly, however, transduction of DMD.48-50 $0^{\mathrm{gE} 51.2}$ myoblasts with $\mathrm{AdV}$.
eCas9.4NLS resulted in significantly higher indel frequencies at $D M D$ exon 51 than those achieved by AdV.Cas9.2NLS (Fig. 6b and Supplementary Fig. 10). 
A

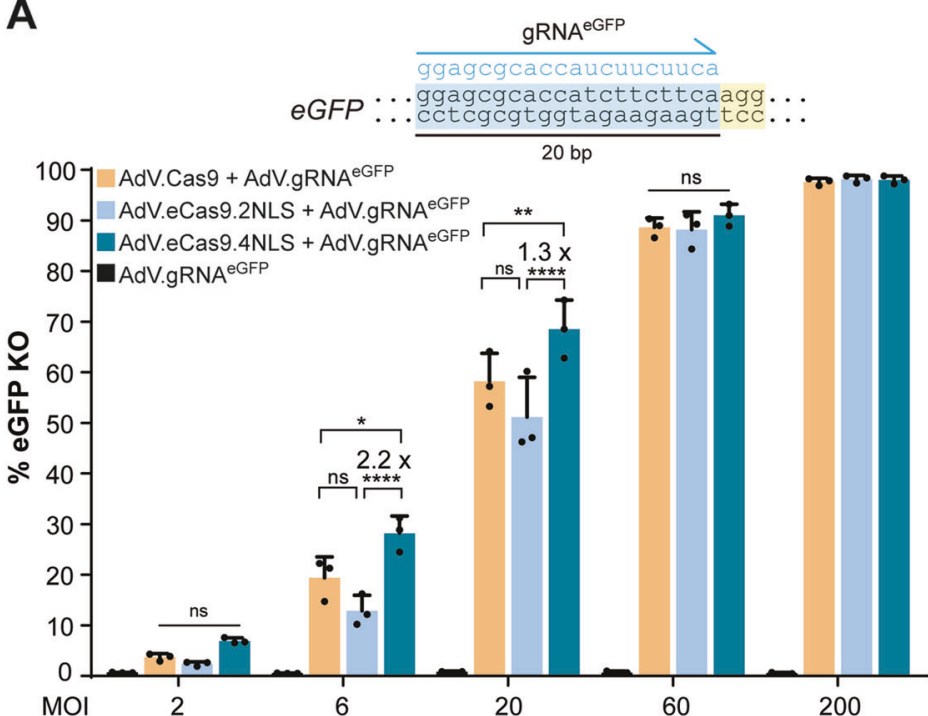

B
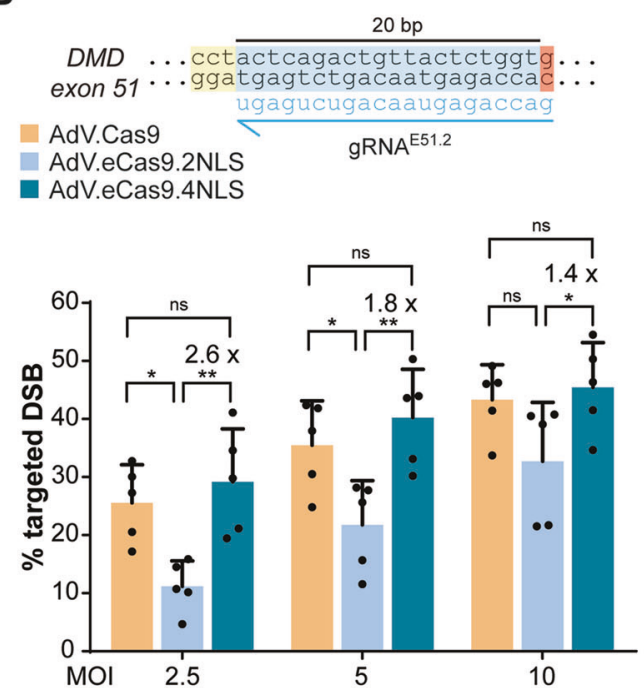

D
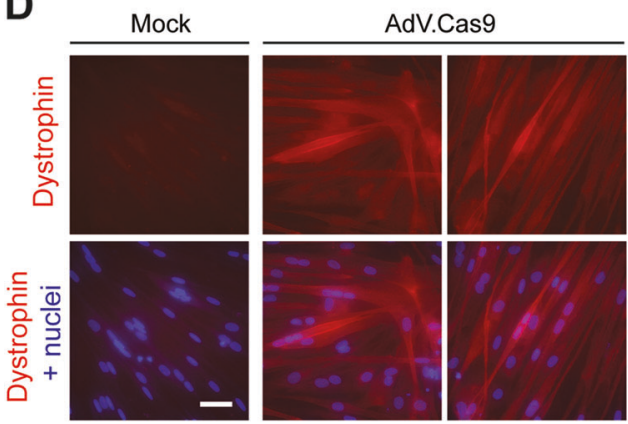

The superior performance of AdV.eCas9.4NLS over AdV.eCas9.2NLS was confirmed in independent experiments targeting $D M D$ exon 53 in DMD.48-50 $0^{\text {gE53 }}$ myoblasts (Fig. 6c and Supplementary Fig. 11). In addition, in DMD. $48-50^{\mathrm{gE} 53}$ myoblasts exposed to the two lowest doses of AdVs, the DSB formation levels achieved by AdV. eCas9.4NLS were significantly higher than those induced
C

AdV.eCas9.2NLS

adV.eCas9.4NLS

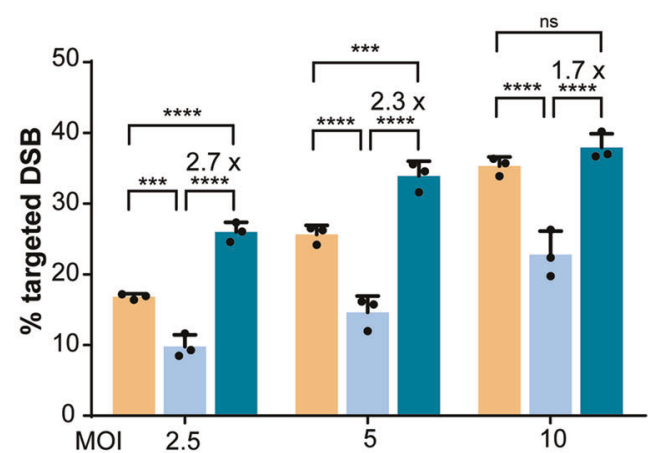

AdV.eCas9.2NLS

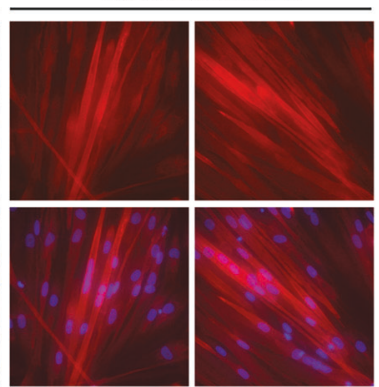

by AdV.Cas9 as well (Fig. 6c and Supplementary Fig. 11). It is also noteworthy mentioning that fourfold higher doses of AdV.eCas9.2NLS were necessary to reach the targeted DSB formation frequencies achieved by AdV.eCas9.4NLS at both $D M D$ target sites (Fig. $6 \mathrm{~b}, \mathrm{c}$ ). In particular, transduction of human myoblasts with AdV.eCas9.4NLS and AdV.eCas9.2NLS at MOIs of 2.5 and $10 \mathrm{IU} / \mathrm{cell}$, 
Fig. 6 Investigating genome editing induced by AdVs encoding standard versus optimized SpCas9 nucleases. a Quantification of $e G F P$ knockout frequencies in AdV-transduced $\mathrm{H} 27$ cells. Upper panel, $e G F P$ target site composed of gRNA-matching sequence (protospacer) and PAM (light blue and yellow boxes, respectively). Lower panel, quantification of target gene knockout by eGFP-directed flow cytometry of $\mathrm{H} 27$ cells transduced with the indicated AdVs. The flow cytometry analysis was performed at 10 days post transduction. Bars and error bars indicate mean and SD, respectively, of three independent experiments. At least 10,000 events, each corresponding to a single viable cell, were measured per sample. b Quantification of targeted DSB formation in patient-derived DMD. $\Delta 48-50^{\text {gE51.2 }}$ myoblasts. DMD. $\triangle 48-50^{\mathrm{gE5} 1.2}$ myoblasts constitutively express gE51.2. Upper panel, target site of gE51.2 addressing the Cas9 nucleases to exon 51 of the DMD locus. The gRNA-matching sequence (protospacer) and PAM are highlighted by the light blue and yellow boxes, respectively. The 1-nt mismatch between gRNA and target site is highlighted in red. Lower panel, DMD. $\Delta 48-50^{\mathrm{gE} 51.2}$ myoblasts were transduced with the indicated $\mathrm{AdVs}$ at different multiplicities of infection (MOIs). Genomic DNA from DMD. $\Delta 48-50^{\text {gE51.2 }}$ myoblasts transduced with the indicated AdVs were subjected to T7EI-based genotyping assays at 3 days post transfection. Negative controls are presented in Supplementary Fig. 10. c Quantification of targeted DSB formation in patient-derived DMD. $445-52^{\mathrm{gE} 53}$ myoblasts. DMD. $\Delta 45-52^{\mathrm{gE} 53}$ myoblasts constitutively express gE53. Upper panel, target site of gE53 targeting SpCas9 nucleases to exon 53 of the DMD locus. The gRNA-matching sequence (protospacer) and PAM are highlighted by the light blue and yellow boxes, respectively. Lower panel, transduction experiments and targeted DNA cleavage analysis were performed as in the experiments done on DMD. $448-50^{\mathrm{gE} 51.2}$ myoblasts. Negative controls are presented in Supplementary Fig. 11. Bars and error bars indicate mean and SD, respectively, of a minimum of three independent experiments. Significance was calculated with two-way ANOVA followed by Tukey's test for multiple comparisons: ns non significant, $P \geq 0.05 ; * P<0.05 ; * * P<0.01 ; * * * P<0.001 ; * * * * P<$ 0.0001. MOI multiplicity of infection. d Dystrophin immunofluorescence microscopy on $D M D$-edited myotubes. Immunostainings for dystrophin were carried out in myotubes differentiated from DMD. $\Delta 48-50^{\mathrm{gE} 51.2}$ myoblasts transduced with the indicated AdVs at an MOI of $10 \mathrm{IU} / \mathrm{cell}$. Mock-transduced myoblasts provided for negative controls. The horizontal white bar corresponds to $50 \mu \mathrm{m}$.

respectively, led to similar frequencies of targeted DSB formation (Fig. 6b, c). These data are relevant in that it opens the perspective for achieving efficient targeted DNA cleavage with substantially lower doses of AdV particles.

Finally, we complemented the $D M D$ gene-editing analyses by performing dystrophin immunofluorescence microscopy on DMD.48-50 $0^{\text {gE51.2 }}$ myotubes differentiated from myoblasts transduced with AdVs encoding the different nucleases (Fig. 6d). The clear detection of dystrophin protein in $D M D$-edited myotubes established that combining AdVmediated gene delivery with optimized SpCas9 nucleases constitute a promising approach for carrying out genome editing in difficult-to-transfect cells (Fig. 6d).

\section{Discussion}

Delivering programmable nucleases into target cell nuclei remains a major challenge for broadening the applicability of
RGN-assisted genome editing. Although linking large prokaryotic Cas9 proteins ( 160 kDa) to NLS motifs is commonly used to drive their nuclear translocation in eukaryotic cells, so far, Cas9 proteins and their variants have been mostly linked to either one or two NLSs [5-7, 17, 47]. In addition, an unbiased quantification of nuclear translocation levels and a thorough assessment of the impact of NLS composition on RGN performance had not been done. To address this, we investigated the effects on nuclear localization and target DNA cleavage of SpCas9 nucleases containing distinct NLS compositions. In these experiments, we deployed a monopartite NLS peptide (PKKKRKV) identified in the SV40 large $\mathrm{T}$ antigen $[48,49]$ and a bipartite NLS (KRPAATKKAGQAKKKK) found in the Xenopus sp. protein nucleoplasmin [50]. These NLS motifs engage the classical NLS-mediated nuclear transport pathway. In brief, SV40 large $\mathrm{T}$ antigen and nucleoplasmin NLSs bind to the cellular importin- $\alpha$ that, after dimerization with importin- $\beta$, mediate the translocation of the attached cargo from the cytoplasm to the nucleus through nuclear pore complexes [24, 25]. In this study, quantitative and unbiased immunofluorescence microscopy assays demonstrated that the eCas9.4NLS nuclease accumulates 2.3-fold more in the nucleus than its parental eCas9.2NLS protein [17].

The experiments performed in HEK.EGFP ${ }^{\text {TetO.KRAB }}$ reporter cells [34, 40, 45] allowed us probing the performance of SpCas9 proteins with different NLS arrangements at target DNA subjected to alternative chromatin conformations. The resulting data suggest that RGNs with eCas9.2NLS or eCas9.4NLS engage with DNA embedded in KRAB-impinged heterochromatin better than Cas9. It will be valuable investigating whether this trend simply results from increased SpCas9 nuclease concentrations in target cell nuclei and/or from local changes in DNA-histone association conferred by the high cationic character of the SpCas9-linked NLS motifs.

We have also validated new combinations of RGN components by demonstrating that high-specificity eCas $9.2 \mathrm{NLS}$ and eCas9.4NLS nucleases are compatible with opt-gRNAs. Importantly, after implementing quantitative off-target detection systems, we gathered data demonstrating that enhanced RGNs, consisting of opt-gRNAs coupled to eCas9.2NLS or eCas9.4NLS, retain DNA cleaving specificities that are higher than that of their Cas 9 counterpart.

A potential limitation of constructs encoding SpCas9 nucleases endowed with various heterologous motifs (e.g., NLSs) and fairly large regulatory elements (e.g., CAG promoter) is that of their delivery into difficult-to-transfect cells. To overcome this hurdle, we generated AdVs encoding Cas9, eCas9.2NLS or eCas9.4NLS. Moreover, we took advantage of the AdV transduction mechanisms to introduce well-defined amounts of different SpCas9 proteins into human cells. We carried out these experiments in 
eGFP-positive HeLa cells and myoblasts isolated from patients with the X-linked muscle-wasting disorder DMD. The eGFP-positive HeLa cells served as a loss-of-function cellular system that allowed for determining gene knockout levels triggered by AdVs encoding Cas9, eCas9.2NLS or eCas9.4NLS. Importantly, exposing target cells to moderate doses of AdV.eCas9.4NLS led to higher eGFP knockout levels than those achieved by using the same doses of AdV. Cas9 or AdV.eCas9.2NLS. The DMD patient-derived myoblasts were instead used as a gain-of-function cellular system based on difficult-to-transfect cells. In this system, RGNs were used to trigger NHEJ-mediated repair of defective $D M D$ reading frames. In these experiments, we found that at matched AdV doses, eCas9.4NLS outperformed eCas 9.2NLS, thus allowing robust $D M D$ reading frame correction. The improved performance of $\mathrm{AdV}$. eCas9.4NLS is expected to be particularly advantageous in experimental settings that require minimizing the exposure of target cells to viral vector components and delivered gene-editing tools.

NLSs have been linked not only to RGNs but also to other types of programmable nucleases, such as zinc finger nucleases and transcription activator-like effector nucleases [51-55]. Of note, when delivering programmable nucleases directly as proteins, NLSs, due to their net positive charge, seem to operate as protein transduction domains hence aiding in cell entry $[52,54,55]$. Thus, these studies could not establish in a strict manner the contribution of NLSs to the nuclear translocation per se due to their simultaneous involvement in cell penetration of programmable nucleases $[52,54,55]$. In the present work, by using AdV-mediated expression of different NLS-linked SpCas9 nucleases we were able to strictly demonstrate that Cas9 engineering profits from including a higher number of NLS sequences than those present in currently used Cas9 nucleases. The impact that delivery methods or experimental settings might have on the activity of Cas9 nucleases endowed with different NLS arrangements and numbers is illustrated in recent studies [56, 57]. For instance, Torres-Ruiz et al. used plasmid transfections to introduce into human cells RGNs containing Cas9 nucleases with a single SV40 NLS at the C-terminus or with this NLS at the C-terminus and a nucleoplasmin NLS at the N-terminus [57]. These experiments did show an enhanced nuclear uptake and DNA cleaving activity by the 2NLS-containing protein in HEK293 cells and human mesenchymal stem cells [57]. Our side-by-side testing of a high-specificity Cas9 variant equally flanked by the SV40 and nucleoplasmin NLSs (i.e., eCas9.2NLS) and its derivative endowed with two extra SV40 NLSs (i.e., eCas9.4NLS) revealed that the latter construct presents superior nuclear uptake and targeted DNA cleaving activities while retaining for the most part the high specificity of its parental, eCas9.2NLS, protein.
Taken these data together, we conclude that designing SpCas9 proteins with extra NLSs is a straightforward and effective strategy to enhance the performance of RGNs and raise the possibility for further improvements via additional optimization of their NLS content. Finally, the testing and use of the resulting optimized gene-editing tools in genetic therapies will profit from their integration with viral vector systems such as the herein applied CD46-targeting AdV platform. This gene delivery platform should permit stoichiometric "all-in-one" delivery of optimized RGN components into therapeutically relevant cell types.

\section{Materials and methods}

\section{Cells}

The human cervix carcinoma HeLa cells (American Type Culture Collection) were cultured in Dulbecco's modified Eagle's medium containing 5\% fetal bovine serum (FBS), at $37^{\circ} \mathrm{C}$ in a $10 \% \mathrm{CO}_{2}$ atmosphere. The origin of and culture conditions for the HeLa cell-derived clone $\mathrm{H} 27$ and H11.7, constitutively expressing eGFP and mTurquoise2, respectively, have been detailed elsewhere [33, 37]. The generation of and the culture conditions for the human embryonic kidney cell-derived cells HEK.EGFP ${ }^{\text {TetO.KRAB }}$ were also detailed elsewhere [40]. The E1- and E2A-complementing AdV packaging cell line PER.E2A was cultured as previously described [28, 58].

Human myoblasts DMD. $\Delta 48-50^{\mathrm{gE} 51.2}$ and DMD. $45-52^{\mathrm{gE} 53}$ were generated and cultured as described in detail elsewhere [46]. DMD. $\Delta 48-50^{\mathrm{g} 551.2}$ myoblasts harbor $D M D$ intragenic deletions spanning exons 48 to 50 and express gRNA gE51.2. DMD.45-52 $2^{\text {gE53 }}$ myoblasts have DMD multiexon deletions spanning exon 45 to 52 and express gRNA gE53. The gE51.2 and gE53 expression units were delivered into the DMD myoblasts by lentiviral vectors encoding a BSD::EGFP marker. After blasticidin selection, the resulting DMD myoblast populations expressed the respective gRNAs [46]. The cells used in this study were mycoplasma free.

\section{Plasmids}

The isogenic constructs AV62_pCAG.Cas9.rBGpA and AW01_pCAG.eSpCas9(1.1).rBGpA, encoding Cas9 and eCas9.2NLS, respectively, were previously described [40]. The former and latter construct harbor, respectively, codonoptimized ORFs coding for the $S$. pyogenes proteins SpCas9 [5], herein dubbed Cas9, and eSpCas9(1.1) [17], herein named eCas9.2NLS. The plasmid encoding eCas9.4NLS was constructed by inserting two NLSs from the SV40 large T antigen, downstream of the ORF coding for eCas9.2NLS. The annotated map and nt sequence of the 
eCas9.4NLS-encoding plasmid are presented in Supplementary Fig. 12. The annotated amino acid sequence of the eCas9.4NLS protein is depicted in Supplementary Fig. 13.

The gRNA acceptor plasmid with the opt-gRNA scaffold AY56_pUC.U6.opt-sgRNA.BveI-stuffer was made by ligating annealed oligonucleotides 5'- GGCCGCACCTGC TGACGTTTCAGAGCTATGCTGGAAACAGCATAGC AAGTTGAAATAAGGCTAGTCCGTTATCAACTTGAA AAAGTGGCACCGAGTCGGTGCTTTTTTTG-3' and 5'AATTCAAAAAAAGCACCGACTCGGTGCCACTTTT TCAAGTTGATAACGGACTAGCCTTATTTCAACTTG CTATGCTGTTTCCAGCATAGCTCTGAAACGTCAGC AGGTGC $-3^{\prime}$ into the NotI/EcoRI-digested construct S7_pUC.U6.sgRNA.BveI-stuffer [40]. The gRNA expressing plasmids harboring standard or opt-gRNA scaffolds were made by ligating a pair of annealed oligonucleotides into the BveI-digested acceptor construct S7_pUC.U6. sgRNA.BveI-stuffer or AY56_pUC.U6.opt-sgRNA.BveIstuffer, respectively. The oligonucleotide pairs used to generate gRNA expression plasmids addressing the Cas9 nuclease to the eGFP.3, eGFP.5, eGFP.6, eGFP.16, and AAVS1 target sites are listed in Supplementary Table 9. The plasmids expressing opt-gRNAs containing different numbers and positions of nt mismatches to a $e G F P$ target site (opt-gOT.1 through opt-gOT.10) were assembled by inserting a pair of annealed oligonucleotides into the BveItreated acceptor construct AY56_pUC.U6.opt-sgRNA. BveI-stuffer. The oligonucleotide pairs used to generate this set of reagents are listed in Supplementary Table 10.

The constructs gRNA_GFP_T2 (Addgene \#41820) and AV59_pg9.20, herein named gRNA ${ }^{\text {eGFP.8 }}$ and gRNA ${ }^{\text {eGFP.9, }}$, respectively, were previously described [34, 38]. The construct pgRNA (Addgene \#41824), herein named gRNA $^{\text {empty }}$, expresses no gRNA and was used as a negative control wherever indicated. The expression of all gRNAs used in this study are under the control of the human U6 promoter whose preferred transcript initiating $\mathrm{nt}$ is a $\mathrm{G}$.

The expression plasmid AM37_pCMV.DsRedEx2.1. bGHpA (DsRed in short) was used as a control for determining the transfection efficiencies in experiments performed in the reporter cells HEK.EGFP ${ }^{\text {TetO.KRAB }}$ [34] and $\mathrm{H} 27$ for determining chromatin impact indexes and specificity profiles of Cas9 nucleases, respectively.

To generate the AdV shuttle plasmid AY37_pAd.Shu. CAG.Cas $9^{\mathrm{eSp}(1.1)}$.rBGpA and AY38_pAd.Shu.CAG. Cas9 $9^{\text {eSp(1.1) }}$.rBGpA.4NLS, the constructs AW01_pCAG. eSpCas9(1.1).rBGpA and eCas9.4NLS were digested with MssI (Thermo Fisher Scientific) and the resulting fragments containing the nuclease expression units were subsequently isolated and ligated into the ScaI restriction sites of AQ08_pAd.Shu.MCS. The map and nt sequence of AQ08_pAd.Shu.MCS plasmid are presented in Supplementary Fig. 14.
Next, the full-length $E 1$ - and E2A-deleted, i.e., secondgeneration, fiber-modified AdV molecular clones AY39_pAd. CAG.Cas $9^{\text {eSp(1.1) }}$.rBGpA. $\Delta$ E2A.F $F^{50}$ and AY40_pAd.CAG. Cas $9^{\text {eSp(1.1) }}$.rBGpA.4NLS. $\Delta$ E2A.F $F^{50}$ were assembled via homologous recombination (HR) after the transformation of E. coli cells BJ5183 ${ }^{\text {pAdEasy-2.50 }}$ [59] with the MssI-treated AY37_pAd.Shu.CAG.Cas9 ${ }^{\text {eSp(1.1) }}$.BGpA and AY38_pAd. Shu.CAG.Cas $9^{\mathrm{eSp}(1.1)}$.rBGpA.4NLS, respectively. The assembly of full-length AdV molecular clones in these bacteria has been detailed elsewhere [59]. Similarly, the fulllength $E 1$ - and E2A-deleted fiber-modified AdV molecular clone AU59_pAd.CAG.Cas9.SV40pA.AE2A.F ${ }^{50}$ was assembled via $\mathrm{HR}$ after the transformation of $E$. coli cells BJ5183 ${ }^{\text {pAdEasy-2.50 }}$ [59] with the MssI-treated AdV shuttle plasmid AU51_pAd.shu.CAG.Cas9.SV40pA. The map and nt sequence of AU51_pAd.shu.CAG.Cas9.SV40pA are presented in Supplementary Fig. 15.

All plasmids were purified with LabNed Plasmid Maxiprep Kit (ImTec Diagnostics) according to the manufacturer's recommendations.

\section{Production and characterization of AdVs}

The constructs AU59_pAd.CAG.Cas9.SV40pA.AE2A.F ${ }^{50}$, AY39_pAd.CAG.Cas $9^{\text {eSp(1.1) }}$.rBGpA. $\Delta$ E2A.F ${ }^{50}$, and AY40_ pAd.CAG.Cas $9^{\mathrm{eSp}(1.1)}$.rBGpA.4NLS. $\Delta$ E2A.F ${ }^{50}$ were used for the production of the fiber-modified, $E 1$ - and $E 2 A$-deleted AdVs AdV.Cas9, AdV.eCas9.2NLS and AdV.eCas9.4NLS, respectively. The protocols used in the generation, purification, and titration of the resulting AdV stocks have been described in detail before [28,60]. The titers of purified AdV.Cas9, AdV.eCas9.2NLS, and AdV.eCas9.4NLS stocks were $2.37 \times 10^{10} \mathrm{IU} / \mathrm{ml}, 13.3 \times 10^{10} \mathrm{IU} / \mathrm{ml}$, and $3.29 \times 10^{10}$ $\mathrm{IU} / \mathrm{ml}$, respectively. The generation and characterization of the fiber-modified, E1- and E2A-deleted, $\mathrm{AdVs} \mathrm{AdV}^{\Delta 2} \mathrm{U} 6$. gRNA $^{\mathrm{S} 1} \cdot \mathrm{F}^{50}$ and $\mathrm{AdV}^{\Delta 2} \mathrm{U} 6 . \mathrm{gRNA}{ }^{\mathrm{GFP}} \cdot \mathrm{F}^{50}$, herein named AdV.gRNA ${ }^{\mathrm{S} 1}$ and AdV.gRNA ${ }^{\mathrm{GFP}}$, respectively, has been described previously [28].

\section{Transfection experiments}

Plasmid transfections for introducing RGN components into human cervix carcinoma cells were initiated by seeding cells in wells of 24-well plates (Greiner Bio-One). HeLa, H27, and H11.7 cells were seeded at a density of $3-4 \times 10^{4}$ cells per well. $24 \mathrm{~h}$ after seeding, the cells were transfected with DNA mixtures consisting of $250 \mathrm{ng}$ of plasmids encoding Cas9 protein and $250 \mathrm{ng}$ of plasmids expressing gRNAs. These DNA mixtures were diluted in $50 \mu \mathrm{l}$ of $150 \mathrm{mM} \mathrm{NaCl}$ (Merck), supplemented with $2.19 \mu \mathrm{l}$ of a $1 \mathrm{mg} / \mathrm{ml}$ polyethylenimine (PEI) solution and directly subjected to vigorous vortexing for about $10 \mathrm{~s}$. After a 15-min incubation at room temperature, the resulting polycation-DNA complexes were 
directly added to the culture medium of the test cells. After a 6-h incubation, the transfection mixtures were replaced with fresh culture medium. At 3 days post transfection, total cellular DNA from HeLa cells was isolated by using the DNeasy Blood \& Tissue kit (Qiagen) following the manufacturer's recommendations. Cultures with transfected $\mathrm{H} 27$ and $\mathrm{H} 11.7$ cells were instead subcultured for 10 days, at which time point, reporter-negative cells were quantified by flow cytometry as described below.

Transfection experiments for comparing the specificity of Cas9, eCas9.2NLS and eCas9.4NLS in H27 cells were done following the scheme provided in Supplementary Table 11 using the PEI-based protocol described in the first paragraph. The expression plasmid AM37_pCMV.DsRedEx2.1. bGHpA [34] was included in each DNA mixture to determine transfection efficiencies at 3 days post transfection. The construct AM51_pgNT [45] provided for a $e G F P$ nontargeting gRNA (gNT) control. The frequencies of $e G F P$ knockout were determined by flow cytometry at 10 days post transfection and were then normalized for the initial transfection efficiencies.

Transfection experiments in HEK.EGFP ${ }^{\text {TetO.KRAB }}$ cells were carried out as detailed elsewhere [40]. In brief, after exposing HEK.EGFP ${ }^{\text {TetO.KRAB }}$ cells to regular growth medium or to medium supplemented with Dox $(500 \mathrm{ng} / \mathrm{ml})$ for $7-10$ days, cells were seeded at a density of $1.5 \times 10^{5}$ cells per well in 24-well plates. The next day, the cells were transfected according to the scheme presented in Supplementary Table 12 using the PEI-based protocol specified in the first paragraph. The expression plasmid AM37_pCMV. DsRedEx2.1.bGHpA was included in the transfection mixtures to evaluate transfection efficiencies by DsRed-direct flow cytometry at 3 days post transfection. Next, the transfected cell cultures were subcultured every 3-4 days for a period of 7 days. In order to activate target transgene expression, at 10 days post transfection HEK.EGFP ${ }^{\text {TetO.KRAB }}$ cells that had not been initially exposed to Dox were subcultured in medium supplemented with Dox $(500 \mathrm{ng} / \mathrm{ml})$ for an additional period of 7 days, after which reporter-directed flow cytometry was used to quantify the $e G F P$ knockout frequencies.

\section{Transduction experiments}

In all experiments that involved cotransductions for introducing RGN components into target cells, the proportion between the MOIs of AdVs encoding SpCas9 proteins and AdVs encoding gRNAs was 1:1. Transduction experiments in $\mathrm{H} 27$ cells were initiated by seeding the cells in wells of 24 -well plates at a density of $4 \times 10^{4}$ cells per well. The next day, the cells were incubated in $500 \mu$ of medium containing AdV particles at the indicated MOIs. At 3 days post transduction inocula were discarded and the cells were subcultured for an additional 7 days. At 10 days post transduction, the presence of eGFP-negative cells was assessed by direct fluorescence microscopy and flow cytometry as described below.

Transduction experiments in HeLa cells were performed as in $\mathrm{H} 27$ cells, except that at 3 days post transduction, the cell cultures were transferred to Millicell EZ SLIDE glass slides (Merck) for performing Xcyto 10 measurements or were harvested for total cellular DNA isolation by using the DNeasy Blood \& Tissue kit (Qiagen).

Gene-editing experiments in DMD. $448-50^{\mathrm{gE} 51.2}$ and DMD.45-52 $2^{\mathrm{gE} 53}$ myoblasts were essentially performed as described elsewhere [46]. In brief, DMD. $448-50^{\text {gE51.2 }}$ and DMD.45-52 $2^{\mathrm{gE} 53}$ myoblasts were seeded at a density of $3 \times$ $10^{4}$ cells per well in 24-well plates (Greiner Bio-One), precoated with a $0.1 \%(\mathrm{w} / \mathrm{v})$ gelatin solution (SigmaAldrich). After $24 \mathrm{~h}$, the cells were incubated in $500 \mu \mathrm{l}$ of medium containing the different amounts of AdV particles. At 3 days post transduction genomic DNA was isolated for assessing the frequencies of DSB formation by T7EI-based genotyping assays (see below) and parallel DMD. $\Delta 48-50^{\text {gE51.2 }}$ myoblast cultures were exposed to mitogenpoor conditions for triggering myogenic differentiation and myotube formation. After 4 days, the myoblasts were stained for dystrophin (see below).

\section{T7EI-based genotyping assays}

The frequencies of indels resulting from targeted DSB formation at the AAVS1 and DMD loci were assessed as follows. Genomic DNA extracted from transfected or transduced target cells was subjected to PCR for amplifying DNA segments spanning the RGN target sites located in AAVS1 and DMD loci. Next, the resulting amplicons were treated with T7EI (New England Biolabs) in the buffer provided by the manufacturer. The primer sequences, the PCR reagents, the cycling parameters and the protocol for the T7EI-based genotyping assays have all been described before [46]. After densitometry of undigested and T7EIdigested amplicons, indel frequencies were calculated by applying the following formula: $100 \times[1-(1-$ fraction (leaved $)^{1 / 2}$ [61].

\section{Flow cytometry}

The frequencies of eGFP-negative and mTurquoise2negative cells in $\mathrm{H} 27$ and $\mathrm{H} 11.7$ cell cultures, respectively, were determined by using a BD LSR II flow cytometer (BD Biosciences). In addition, DsRed-directed flow cytometry was used for determining transfection efficiencies in HEK. EGFP $^{\text {TetO.KRAB }}$ reporter cells. At least 10,000 viable single cells were analyzed per experimental condition. Data were analyzed with the aid of FlowJo 10.4 software (Tree Star). 
Mock-transduced cells served to establish the cutoff between reporter-positive and reporter-negative cell populations.

\section{Direct fluorescence microscopy}

Targeted $e G F P$ knockout in AdV-transduced $\mathrm{H} 27$ cell cultures was monitored by direct fluorescence microscopy. The $\mathrm{H} 27$ cell nuclei were stained by adding $10 \mu \mathrm{g} / \mathrm{ml}$ Hoechst 33342 (Molecular Probes) for $10 \mathrm{~min}$. After washing twice with phosphate-buffered saline (PBS), $\mathrm{pH}$ 7.4 , culture medium was added to the cell cultures. The eGFP- and Hoechst 33342-specific signals were detected by using an AF6000 LX system and LAS AF software version 2.7.4.10100 (both from Leica).

\section{Assessment of Cas9 nuclear expression with Xcyto 10}

HeLa cells were transfected or transduced as indicated above. Three days later, HeLa cells were seeded in Millicell EZ SLIDE glass (8-well or 4-well, Merck) and were kept in regular growth medium. After overnight incubation at $37^{\circ} \mathrm{C}$ in a $10 \% \mathrm{CO}_{2}$ atmosphere, the HeLa cells were fixed with $4 \%$ (w/v) paraformaldehyde in PBS for $15 \mathrm{~min}$ at $4{ }^{\circ} \mathrm{C}$. After removing the fixative by two washes with PBS, the cells were permeabilized by adding $0.25 \%$ (v/v) Triton X-100 (SigmaAldrich) in PBS for $10 \mathrm{~min}$ at room temperature. The detergent was removed by three 5-min washes with TBST consisting of $0.1 \mathrm{M}$ Tris- $\mathrm{HCl}(\mathrm{pH} 7.5), 0.15 \mathrm{M} \mathrm{NaCl}$, and $0.05 \%$ Tween-20 (Merck). Next, a blocking step was performed by incubating the cells at room temperature for $10 \mathrm{~min}$ with TNB containing $0.1 \mathrm{M}$ Tris- $\mathrm{HCl}(\mathrm{pH} 7.5), 0.15 \mathrm{M} \mathrm{NaCl}$, and $0.5 \%$ blocking reagent for nucleic acid hybridization and detection (Roche). A mouse monoclonal IgG1 antibody directed against the SpCas9 protein (ab191468; Abcam) was diluted 1:200 in TNB and was incubated with the cells for $1 \mathrm{~h}$ at room temperature. Next, the cells were rinsed by three 5 -min washes with TBST. The cells were then incubated for $1 \mathrm{~h}$ at room temperature in the dark with an Alexa Fluor 488 anti-Mouse $\operatorname{IgG}(\mathrm{H}+\mathrm{L}$; A11001 Invitrogen) diluted 1:500 in TNB. Excess of secondary antibody was removed by three 5-min washes with TBST. After washing once with PBS, the cells were stained with BlueMask-1 (Solutions $12+21$, Chemometec) diluted 1:1000 in PBS for $30 \mathrm{~min}$ in the dark. After removing the Bluemask-1 dye via four washes with PBS, the chambers were disassembled, gently washed in distilled water, and let to air dry. Glass slides were embedded in $70 \mu \mathrm{l}$ of Prolong Gold with DAPI (Thermo Fisher Scientific) and were sealed with a glass coverslip. Digital images were acquired by using Xcyto 10 Quantitative Cell Imager (Chemometec) with automated segmentation done by XcytoView 1.0.63.0 software based on the DAPI and BlueMask-1 staining. Finally, the data were analyzed with the aid of FlowJo 10.4 software (Tree Star).

\section{Immunofluorescence microscopy}

HeLa cells were transfected as indicated in "Transfection experiments" section. Three days post transfection, HeLa cells were fixed with $4 \%(\mathrm{w} / \mathrm{v})$ paraformaldehyde in PBS for $30 \mathrm{~min}$ at room temperature. Next, the HeLa cells were stained as described elsewhere [46], except for the following variations. A mouse monoclonal $\mathrm{IgG} 1$ antibody directed against the SpCas9 protein (ab191468; Abcam) was diluted 1:200 in $10 \mathrm{mM}$ glycine in PBS (PBSG) with 5\% FBS and was then added to the cells for overnight incubation at $4{ }^{\circ} \mathrm{C}$. Subsequently, the cells were stained with an Alexa Fluor 488 anti-Mouse $\operatorname{IgG}(\mathrm{H}+\mathrm{L}$; A11001 Invitrogen) diluted 1:500 in PBSG containing 5\% FBS. Digital images were acquired by using an AF6000 LX system and LAS AF software version 2.7.4.10100 (both from Leica).

DMD. $\Delta 48-50^{\mathrm{gE} 51.2}$ myoblasts were transduced as described in "Transduction experiments" section. Three days post transduction, cell populations were exposed to mitogen-poor conditions for triggering myotube differentiation. The composition of the differentiation medium is specified elsewhere [46]. After 4 days, the resulting myotubes were fixed with $4 \%(\mathrm{w} / \mathrm{v})$ paraformaldehyde in PBS for $10 \mathrm{~min}$ at $4{ }^{\circ} \mathrm{C}$ and were permeabilized by adding $0.5 \%(\mathrm{v} / \mathrm{v})$ Triton $\mathrm{X}-100$ in TBS $(0.05 \mathrm{M}$ Tris-HCl, $\mathrm{pH} 7.5$ and $0.1 \mathrm{M} \mathrm{NaCl})$ for $10 \mathrm{~min}$ at room temperature. After three 5-min washes with TBST, a blocking step was performed by incubating the cells for $\sim 2 \mathrm{~h}$ in TBS supplemented with $0.1 \%$ (v/v) Triton X-100, 2\% (w/ v) BSA (Sigma-Aldrich) and $0.1 \%$ azide (Merck) at room temperature. A rabbit polyclonal antibody specific for the Cterminus of dystrophin (ab15277; Abcam) was diluted 1:100 in TBS supplemented with $0.1 \%(\mathrm{v} / \mathrm{v})$ Triton X-100, $2 \%$ $(\mathrm{w} / \mathrm{v}) \mathrm{BSA}$ and $0.1 \%$ azide. After removing the blocking solution, the cells were incubated overnight at $4{ }^{\circ} \mathrm{C}$ with the antibody. Next, the primary antibody was removed by three 5-min washes with TBST and the secondary antibody Alexa Fluor 568 anti-rabbit $\operatorname{IgG}(\mathrm{H}+\mathrm{L}$; A11036 Invitrogen) was added to the cells diluted 1:500 in TBS supplemented with $0.1 \%(\mathrm{v} / \mathrm{v})$ Triton X-100, $2 \%(\mathrm{w} / \mathrm{v})$ BSA and $0.1 \%$ azide for $45 \mathrm{~min}$ at room temperature. After three 5-min washes with TBST, the cells were incubate with Hoechst 33342 (Molecular Probes) diluted 1:1000 in PBS for $10 \mathrm{~min}$ at room temperature. The DNA dye was subsequently removed by three washes with PBS. Digital images were acquired with AF6000 LX system and LAS AF software version 2.7.4.10100 (both from Leica).

\section{Statistical analyses}

Data derived from a minimum of three independent biological replicates were analyzed by using GraphPad Prism 8.0.1 software. Statistical significance was calculated with the tests indicated in the various figure legends. $P$ values 
lower than 0.05 were considered to be statistically significant. No statistical methods were used to predefine sample size and no data were excluded from the analyses. There was no use of randomization methods for sample allocation to experimental groups and the investigators were not blinded to group allocation. The variance, including SD and SEM values, resulting from statistically compared experimental groups were similar.

\section{Data availability}

All relevant results generated in this study are available within the paper and accompanying Supplementary Information.

Acknowledgements The authors thank Rene McLaughlin and Edwin de Haas (both from the Flow Cytometry Facility, Leiden University Medical Center, Leiden, The Netherlands) for their technical assistance in setting up the experiments with Xcyto 10 Quantitative Cell Imager. The authors also thank Marcella Dias Brescia (Department of Cell and Chemical Biology, Leiden University Medical Center, Leiden, The Netherlands) for her support with the immunofluorescence microscopy assays in the human myoblasts.

Funding This work was supported by the Departments of Pediatrics/ Willem-Alexander Kinderziekenhuis, Cell and Chemical Biology and Human Genetics from the Leiden University Medical Center, Leiden, The Netherlands. This work was also supported by research grants from the Dutch Prinses Beatrix Spierfonds (W.OR16-13) and the Dutch Duchenne Parent Project (DPP/NL) (to MAFVG).

Author contributions IM and MAFVG conceived and initiated the research; SMvdM, ACL, RCH, and MAFVG directed the research; IM, MAFVG, and RCH designed the experiments; IM, JL, and JMJ generated and characterized reagents and tools; IM performed all the experiments except those carried out in human myoblasts, which were instead performed by HAZ and ITO; and those assessing Cas9 nuclease specificities with the set of ten opt-gRNAs (opt-gOT.1-optgOT.10), which were carried out by HAZ; QW designed, constructed, and validated the opt-gOT.1-opt-gOT.10 gRNA plasmid set. IM, MAFVG and HAZ analyzed the data together with all authors; IM wrote the manuscript with the aid of MAFVG. All the authors read and approved the manuscript.

\section{Compliance with ethical standards}

Conflict of interest The authors declare that they have no conflict of interest.

Publisher's note Springer Nature remains neutral with regard to jurisdictional claims in published maps and institutional affiliations.

Open Access This article is licensed under a Creative Commons Attribution 4.0 International License, which permits use, sharing, adaptation, distribution and reproduction in any medium or format, as long as you give appropriate credit to the original author(s) and the source, provide a link to the Creative Commons license, and indicate if changes were made. The images or other third party material in this article are included in the article's Creative Commons license, unless indicated otherwise in a credit line to the material. If material is not included in the article's Creative Commons license and your intended use is not permitted by statutory regulation or exceeds the permitted use, you will need to obtain permission directly from the copyright holder. To view a copy of this license, visit http://creativecommons. org/licenses/by/4.0/.

\section{References}

1. Doudna JA, Charpentier E. The new frontier of genome engineering with CRISPR-Cas9. Science. 2014;346:1258096.

2. Maggio I, Gonçalves MA. Genome editing at the crossroads of delivery, specificity, and fidelity. Trends Biotechnol. 2015;33:280-91.

3. Murugan K, Babu K, Sundaresan R, Rajan R, Sashital DG. The revolution continues: newly discovered systems expand the CRISPR-Cas toolkit. Mol Cell. 2017;68:15-25.

4. Chen X, Gonçalves MA. DNA, RNA, and protein tools for editing the genetic information in human cells. iScience. 2018;6:247-63.

5. Mali P, Yang L, Esvelt KM, Aach J, Guell M, DiCarlo JE, et al. RNA-guided human genome engineering via Cas9. Science. 2013;339:823-6.

6. Jinek M, East A, Cheng A, Lin S, Ma E, Doudna J. RNAprogrammed genome editing in human cells. Elife. 2013;2:e00471.

7. Cong L, Ran FA, Cox D, Lin S, Barretto R, Habib N, et al. Multiplex genome engineering using CRISPR/Cas systems. Science. 2013;339:819-23.

8. Nishimasu H, Ran FA, Hsu PD, Konermann S, Shehata SI, Dohmae N, et al. Crystal structure of Cas9 in complex with guide RNA and target DNA. Cell. 2014;156:935-49.

9. Jinek M, Jiang F, Taylor DW, Sternberg SH, Kaya E, Ma E, et al. Structures of Cas9 endonucleases reveal RNA-mediated conformational activation. Science. 2014;343:1247997.

10. Anders C, Niewoehner O, Duerst A, Jinek M. Structural basis of PAM-dependent target DNA recognition by the Cas9 endonuclease. Nature. 2014;513:569-73.

11. Jinek M, Chylinski K, Fonfara I, Hauer M, Doudna JA, Charpentier $\mathrm{E}$, et al. A programmable dual-RNA-guided DNA endonuclease in adaptive bacterial immunity. Science. 2012;337:816-21.

12. Briner AE, Donohoue PD, Gomaa AA, Selle K, Slorach EM, Nye $\mathrm{CH}$, et al. Guide RNA functional modules direct Cas9 activity and orthogonality. Mol Cell. 2014;56:333-9.

13. Kleinstiver BP, Prew MS, Tsai SQ, Topkar VV, Nguyen NT, Zheng Z, et al. Engineered CRISPR-Cas9 nucleases with altered PAM specificities. Nature. 2015;523:481-5.

14. Hu JH, Miller SM, Geurts MH, Tang W, Chen L, Sun N, et al. Evolved Cas9 variants with broad PAM compatibility and high DNA specificity. Nature. 2018;556:57-63.

15. Kleinstiver BP, Pattanayak V, Prew MS, Tsai SQ, Nguyen NT, Zheng Z, et al. High-fidelity CRISPR-Cas9 nucleases with no detectable genome-wide off-target effects. Nature. 2016;529:490-5.

16. Chen JS, Dagdas YS, Kleinstiver BP, Welch MM, Sousa AA, Harrington LB, et al. Enhanced proofreading governs CRISPRCas9 targeting accuracy. Nature. 2017;550:407-10.

17. Slaymaker IM, Gao L, Zetsche B, Scott DA, Yan WX, Zhang F, et al. Rationally engineered Cas 9 nucleases with improved specificity. Science. 2016;351:84-8.

18. Casini A, Olivieri M, Petris G, Montagna C, Reginato G, Maule $\mathrm{G}$, et al. A highly specific SpCas9 variant is identified by in vivo screening in yeast. Nat Biotechnol. 2018;36:265-71.

19. Lee JK, Jeong E, Lee J, Jung M, Shin E, Kim YH, et al. Directed evolution of CRISPR-Cas9 to increase its specificity. Nat Commun. 2018;9:3048.

20. Fu Y, Sander JD, Reyon D, Cascio VM, Joung JK. Improving CRISPR-Cas nuclease specificity using truncated guide RNAs. Nat Biotechnol. 2014;32:279-84.

21. Chen B, Gilbert LA, Cimini BA, Schnitzbauer J, Zhang W, Li $\mathrm{GW}$, et al. Dynamic imaging of genomic loci in living human cells 
by an optimized CRISPR/Cas system. Cell. 2013;155:1479-91. Erratum in: Cell. 2014;156:373

22. Dang Y, Jia G, Choi J, Ma H, Anaya E, Ye C, et al. Optimizing sgRNA structure to improve CRISPR-Cas9 knockout efficiency. Genome Biol. 2015;16:280.

23. Glass Z, Lee M, Li Y, Xu Q. Engineering the delivery system for CRISPR-based genome editing. Trends Biotechnol. 2018;36:173-85.

24. Christie M, Chang CW, Róna G, Smith KM, Stewart AG, Takeda AA, et al. Structural biology and regulation of protein import into the nucleus. J Mol Biol. 2016;428:2060-90.

25. Sun Y, Xian L, Xing H, Yu J, Yang Z, Yang T, et al. Factors influencing the nuclear targeting ability of nuclear localization signals. J Drug Target. 2016;24:927-33.

26. Gonçalves MA, de Vries AA. Adenovirus: from foe to friend. Rev Med Virol. 2006;16:167-86.

27. Chen X, Gonçalves MA. Engineered viruses as genome editing devices. Mol Ther. 2016;24:447-57.

28. Maggio I, Holkers M, Liu J, Janssen JM, Chen X, Gonçalves MA. Adenoviral vector delivery of RNA-guided CRISPR/Cas9 nuclease complexes induces targeted mutagenesis in a diverse array of human cells. Sci Rep. 2014;4:5105.

29. Xu L, Park KH, Zhao L, Xu J, El Refaey M, Gao Y, et al. CRISPR-mediated genome editing restores dystrophin expression and function in mdx mice. Mol Ther. 2016;24:564-9.

30. Maggio I, Liu J, Janssen JM, Chen X, Gonçalves MA. Adenoviral vectors encoding CRISPR/Cas9 multiplexes rescue dystrophin synthesis in unselected populations of DMD muscle cells. Sci Rep. 2016;6:37051.

31. Nielsen S, Yuzenkova Y, Zenkin N. Mechanism of eukaryotic RNA polymerase III transcription termination. Science. 2013;340:1577-80.

32. Papapetrou EP, Schambach A. Gene insertion into genomic safe harbors for human gene therapy. Mol Ther. 2016;24:678-84.

33. Gonçalves MA, van der Velde I, Knaän-Shanzer S, Valerio D, de Vries AA. Stable transduction of large DNA by high-capacity adeno-associated virus/adenovirus hybrid vectors. Virology. 2004; 321:287-96.

34. Chen X, Liu J, Janssen JM, Gonçalves MA. The chromatin structure differentially impacts high-specificity CRISPR-Cas9 nuclease strategies. Mol Ther Nucleic Acids. 2017;8:558-63.

35. Zhang D, Zhang H, Li T, Chen K, Qiu JL, Gao C. Perfectly matched 20-nucleotide guide RNA sequences enable robust genome editing using high-fidelity SpCas9 nucleases. Genome Biol. 2017;18:191.

36. Kim S, Bae T, Hwang J, Kim JS. Rescue of high-specificity Cas 9 variants using sgRNAs with matched 5 ' nucleotides. Genome Biol. 2017; $18: 218$.

37. Chen X, Janssen JM, Liu J, Maggio I, 't Jong AEJ, Mikkers HMM, et al. In trans paired nicking triggers seamless genome editing without double-stranded DNA cutting. Nat Commun. 2017;8:657.

38. Mali P, Aach J, Stranges PB, Esvelt KM, Moosburner M, Kosuri $\mathrm{S}$, et al. CAS9 transcriptional activators for target specificity screening and paired nickases for cooperative genome engineering. Nat Biotechnol. 2013;31:833-8.

39. Hsu PD, Scott DA, Weinstein JA, Ran FA, Konermann S, Agarwala V, et al. DNA targeting specificity of RNA-guided Cas9 nucleases. Nat Biotechnol. 2013;31:827-32.

40. Chen X, Rinsma M, Janssen JM, Liu J, Maggio I, Gonçalves MA. Probing the impact of chromatin conformation on genome editing tools. Nucleic Acids Res. 2016;44:6482-92.

41. Wu X, Scott DA, Kriz AJ, Chiu AC, Hsu PD, Dadon DB, et al. Genome-wide binding of the CRISPR endonuclease Cas9 in mammalian cells. Nat Biotechnol. 2014;32:670-6.

42. Knight SC, Xie L, Deng W, Guglielmi B, Witkowsky LB, Bosanac L, et al. Dynamics of CRISPR-Cas9 genome interrogation in living cells. Science. 2015;350:823-6.
43. Daer RM, Cutts JP, Brafman DA, Haynes KA. The impact of chromatin dynamics on Cas9-mediated genome editing in human cells. ACS Synth Biol. 2017;6:428-38.

44. Jensen KT, Fløe L, Petersen TS, Huang J, Xu F, Bolund L, et al. Chromatin accessibility and guide sequence secondary structure affect CRISPR-Cas9 gene editing efficiency. FEBS Lett. 2017; 591:1892-901.

45. Janssen JM, Chen X, Liu J, Gonçalves MAFV. The chromatin structure of CRISPR-Cas9 target DNA controls the balance between mutagenic and homology-directed gene-editing events. Mol Ther Nucleic Acids. 2019;16:141-54.

46. Maggio I, Stefanucci L, Janssen JM, Liu J, Chen X, Mouly V, et al. Selection-free gene repair after adenoviral vector transduction of designer nucleases: rescue of dystrophin synthesis in DMD muscle cell populations. Nucleic Acids Res. 2016;44:1449-70.

47. Zafra MP, Schatoff EM, Katti A, Foronda M, Breinig M, Schweitzer AY, et al. Optimized base editors enable efficient editing in cells, organoids and mice. Nat Biotechnol. 2018;36:888-93.

48. Kalderon D, Richardson WD, Markham AF, Smith AE. Sequence requirements for nuclear location of simian virus 40 large-T antigen. Nature. 1984;311:33-8.

49. Kalderon D, Roberts BL, Richardson WD, Smith AE. A short amino acid sequence able to specify nuclear location. Cell. 1984; 39:499-509.

50. Dingwall C, Sharnick SV, Laskey RA. A polypeptide domain that specifies migration of nucleoplasmin into the nucleus. Cell. 1982;30:449-58.

51. Gaj T, Gersbach CA, Barbas CF 3rd. ZFN, TALEN, and CRISPR/ Cas-based methods for genome engineering. Trends Biotechnol. 2013;31:397-405.

52. Liu J, Gaj T, Wallen MC, Barbas CF. Improved cell-penetrating zinc-finger nuclease proteins for precision genome engineering. Mol Ther Nucleic Acids. 2015;4:e232.

53. Ru R, Yao Y, Yu S, Yin B, Xu W, Zhao S, et al. Targeted genome engineering in human induced pluripotent stem cells by penetrating TALENs. Cell Regen. 2013;2:5.

54. Liu J, Gaj T, Yang Y, Wang N, Shui S, Kim S, et al. Efficient delivery of nuclease proteins for genome editing in human stem cells and primary cells. Nat Protoc. 2015;10:1842-59.

55. Staahl BT, Benekareddy M, Coulon-Bainier C, Banfal AA, Floor $\mathrm{SN}$, Sabo JK, et al. Efficient genome editing in the mouse brain by local delivery of engineered Cas 9 ribonucleoprotein complexes. Nat Biotechnol. 2017;35:431-4.

56. Hu P, Zhao X, Zhang Q, Li W, Zu Y. Comparison of various nuclear localization signal-fused Cas9 Proteins and Cas9 mRNA for genome editing in zebrafish. G3. 2018;8:823-31.

57. Torres-Ruiz R, Martinez-Lage M, Martin MC, Garcia A, Bueno C, Castaño J, Ramirez JC, et al. Efficient recreation of $\mathrm{t}(11 ; 22)$ EWSR1-FLI1+ in human stem cells using CRISPR/Cas9. Stem Cell Rep. 2017;8:1408-20.

58. Havenga MJ, Holterman L, Melis I, Smits S, Kaspers J, Heemskerk E, et al. Serum-free transient protein production system based on adenoviral vector and PER.C6 technology: high yield and preserved bioactivity. Biotechnol Bioeng. 2008;100:273-83.

59. Janssen JM, Liu J, Skokan J, Gonçalves MA, de Vries AA. Development of an AdEasy-based system to produce first- and second-generation adenoviral vectors with tropism for CAR- or CD46-positive cells. J Gene Med. 2013;15:1-11.

60. Holkers M, Cathomen T, Gonçalves MA. Construction and characterization of adenoviral vectors for the delivery of TALENs into human cells. Methods. 2014;69:179-87.

61. Miller JC, Holmes MC, Wang J, Guschin DY, Lee YL, Rupniewski I, et al. An improved zinc-finger nuclease architecture for highly specific genome editing. Nat Biotechnol. 2007;25:778-85. 